\title{
Air evacuation during vacuum bag only prepreg processing of honeycomb sandwich structures: in-plane air extraction prior to cure
}

\author{
Arthur Levy, James Kratz, Pascal Hubert*
}

Department of Mechanical Engineering, McGill University, 817 Sherbrooke St. West, Montréal QC, H3A 0C3, Canada.

\begin{abstract}
Out-of-autoclave (OoA) prepregs offer good in-plane air permeability that enable efficient in-plane airextraction. In this paper, OoA prepregs skins were applied to honeycomb sandwich structures, to act as in-plane air extraction media during the vacuum hold prior to cure. An in-plane extraction model was developed to predict the pressure drop in a honeycomb sandwich structure. The model was experimentally validated by measuring the local pressure in a one meter long sandwich structure. The model provides design guidelines vacuum hold duration to achieve a given final pressure in the part prior to cure. Finally, the in-plane and transverse extraction strategies were compared for large parts. The in-plane extraction was faster for parts smaller than $0.4 \mathrm{~m}$.
\end{abstract}

Keywords: A. Honeycomb, C. Analytical modelling, E. Prepreg, E. Consolidation

\section{Introduction}

Out-of-autoclave (OoA) prepreg processing offers end users familiar prepreg lay-up techniques and part quality without the size constraints and high investment required for autoclave processing. However, the consolidation pressure is lower because laminates are cured in an atmospheric pressure oven, under vacuumbag-only (VBO) processing conditions. Therefore, the maximum consolidation pressure available to oppose or dissolve volatiles in VBO manufacturing is limited to 1 atmosphere $\left(1.013 \times 10^{5} \mathrm{~Pa}\right)$, significantly less than traditional autoclave prepreg processing $\left(7 \times 10^{5} \mathrm{~Pa}\right)$. Similar to other low-pressure manufacturing processes, the critical first step in VBO processing is to evacuate air from the laminate to reduce as many entrapped volatiles as possible, in-order to minimize final part porosity [1]. This is accomplished by holding the part under vacuum for an extended period of time before initiating the elevated temperature cure [2].

Some general recommendations have been offered to determine the length of the vacuum hold. For parts smaller than $0.6 \mathrm{~m} \times 0.6 \mathrm{~m}$ with a uniform thickness, a $4 \mathrm{~h}$ vacuum hold is suggested by the material supplier,

\footnotetext{
*Corresponding author

Email addresses: arthur.levy@mcgill.ca (Arthur Levy), james.kratz@mail.mcgill.ca (James Kratz), pascal.hubert@mcgill.ca (Pascal Hubert) 
and larger or more complicated lay-ups may require a vacuum hold of at least $16 \mathrm{~h}$ prior to cure [3]. Clearly, some trial and error is needed to refine the duration of the vacuum hold for a given lay-up, but better guidelines are needed to avoid excessive vacuum hold times while ensuring the majority of entrapped air is removed.

The first step in estimating the vacuum hold time is to measure the air permeability of the prepreg in-order to understand the flow capacity of the lay-up. Since composite materials are anisotropic, the air permeability tensor is needed to predict the evacuation behaviour [4]. The in-plane (X-Y) air permeability is often two orders of magnitude higher than the transverse $(Z)$ air permeability for out-of-autoclave (OoA) prepreg materials [5-9]. This anisotropy between the in-plane and out of plane permeabilities of the OoA prepreg systems is caused by the resin prepregging techniques. The prepregging process partially saturates the fibre reinforcement with resin, creating central dry fibre regions within the tow bundles [10]. As a result, the dry fibre tows create porous regions within the centre of each prepreg ply with a high cross-sectional area for fluid flow.

Once the air permeability of the prepreg is known, models could be useful to predict the evacuation of entrapped air. Process models that have been successfully used to understand and mitigate processinduced defects in autoclave and liquid moulding applications can be applied to VBO processing [11]. The influence of temperature and pressure during the processing cycle can be used to minimize void formation and growth, reduce temperature gradients and exotherms, maximize fibre volume fraction, improve dimensional stability, and ensure a fully saturated pore space between fibres before the resin gels [11-13]. The physical laws, constitutive equations, and boundary conditions in VBO processing can be used to predict the gas pressure distribution within an OoA prepreg lay-up as a function of space and time.

In the literature, separate studies have investigated the in-plane and through-thickness gas flow in OoA prepregs. One notable study developed a 1D air evacuation model for monolithic prepreg laminates in-order to predict the vacuum hold times needed to evacuate a given mass of entrapped air [5].

In the case of OoA processing of a honeycomb sandwich panel, a large amount of air is entrapped in the core that acts as a reservoir. Therefore, the time required to evacuate this entrapped air is increased when compared to laminates. Still, most of this air has to be evacuated prior to heating to prevent voids in the skins [1]. The through-thickness air permeability of honeycomb skins, and the corresponding throughthickness air evacuation from the honeycomb core has been thoroughly investigated [14-17]. It consists in using a perforated peel ply and allows for air extraction on the whole skin face, thus ensuring an air flow through the thickness of the skin. On the contrary, the in-plane air evacuation of honeycomb structures has not been addressed in the literature. It consists in using edge breathing strategy around the skin's perimeter, thus making use of the higher in-plane permeability of the system. Therefore, no comparison is available to identify which air extraction technique is more efficient prior to cure.

The proposed prepreg air evacuation models have yet to be validated on larger scale parts because 
the experimental complexity of measuring the gas pressure distribution or rate of extraction at multiple locations within a prepreg lay-up. Air evacuation models for honeycomb structures are more realistic to validate because the space within a honeycomb cell can accommodate a pressure measuring device. Miniature pressures sensors have been used to measure resin pressure in composite processing [18], and could be used to measure the gas pressure distribution within honeycomb panels. In this work, a model was developed to predict the in-plane extraction of entrapped air from honeycomb panels featuring OoA prepreg skins and a central embedded honeycomb core. The governing equations were solved using a finite element method, and validated by placing miniature pressure sensors within the honeycomb core. The measured pressure response within the panel was compared to model predictions during the vacuum hold prior to cure.

\section{Modelling}

The sandwich structure considered in this work featured two OoA prepreg skins and an embedded honeycomb core (see Figure 1). In-plane evacuation was considered to be the only air extraction mechanism for the part. Through skin thickness air and resin flow was prevented in the upper skin by an impermeable release film, and the lower skin was placed against a metallic tool.

\subsection{Constitutive Model}

\subsubsection{Flow in the porous skin}

The semi-impregnated materials behave as a porous media, and Darcy's law was used to describe the air flow within the prepreg skin $[9,11,14,19]$ :

$$
\frac{\boldsymbol{K}}{\mu} \nabla P=-\boldsymbol{v}
$$

where $\boldsymbol{K}$ is the permeability of the skin, $P$ is the pressure, $\boldsymbol{v}$ the apparent velocity, and $\mu$ the air viscosity. $\boldsymbol{K}$ is a tensorial property since the fabric is an anisotropic medium. In the principal directions $(x, y, z)$,

$$
\boldsymbol{K}=\left[\begin{array}{ccc}
K_{x} & 0 & 0 \\
0 & K_{y} & 0 \\
0 & 0 & K_{z}
\end{array}\right]_{(x, y, z)}
$$

where $K_{x}$ and $K_{y}$ are the in-plane air permeabilities and $K_{z}$ the transverse.

Wu and Pruess [20] suggested that the Klinkenberg effect [21] may modify Eq. (1) as gas molecules may slip at the interface of the solid fibres, resulting in a non-zero fluid velocity. This deviation from laminar flow theory may overestimate the gas flow velocity at low pressure. This effect becomes non-negligible when the pore size is very small compared to the mean free path of the gas molecule. In the case of composite fabrics, the characteristic pore size is a few microns (typical carbon fibre diameter of $7 \mu \mathrm{m}$ ); and under vacuum processing (around $10^{4} \mathrm{~Pa}$ ), the air mean free path is about $0.1 \mu \mathrm{m}$. Therefore, the Klinkenberg effect may 
influence the intrinsic permeability values. However, if the permeability is characterized for gas flow, using techniques close to vacuum bag processing conditions, the measured value is the effective permeability that includes Klinkenberg effects [22].

Some skin consolidation will occur during the first 30-60 minutes of vacuum application [9], however, after this initial period, the fibre and matrix can be considered as a rigid bed and the conservation of mass in the skin reduces to the air phase. The continuity equation for the skin becomes [23]:

$$
\boldsymbol{\nabla} \cdot\left(\rho_{a} \boldsymbol{v}\right)=-\phi \frac{\partial \rho_{a}}{\partial t}
$$

where $\rho_{a}$ is the density of air and $\phi$ the porosity of the skin. Using the ideal gas equation of state under isothermal conditions, the density of air $\rho_{a}$ is proportional to the pressure $P$ [24]. Equation (3) then becomes:

$$
\boldsymbol{\nabla} \cdot(P \boldsymbol{v})=-\phi \frac{\partial P}{\partial t} .
$$

Using Eq. (1), the equation describing flow in the porous medium is obtained [9, 14, 19, 24-26]:

$$
\boldsymbol{\nabla} \cdot\left(P \frac{\boldsymbol{K}}{\mu} \nabla P\right)=\phi \frac{\partial P}{\partial t}
$$

Considering a two dimensional problem with a basis $(X, Z)$ in the principal direction of $\boldsymbol{K}$ such that:

$$
\boldsymbol{K}=\left[\begin{array}{cc}
K_{x} & 0 \\
0 & K_{z}
\end{array}\right]_{(X, Z)}
$$

with a uniform permeability and viscosity, Eq. (5) becomes:

$$
\frac{K_{x}}{\mu} \frac{\partial\left(P \frac{\partial P}{\partial x}\right)}{\partial x}+\frac{K_{z}}{\mu} \frac{\partial\left(P \frac{\partial P}{\partial z}\right)}{\partial z}=\phi \frac{\partial P}{\partial t}
$$

or

$$
\underbrace{\frac{K_{x}}{2 \mu} \frac{\partial^{2}\left(P^{2}\right)}{\partial x^{2}}}_{A_{x}}+\underbrace{\frac{K_{z}}{2 \mu} \frac{\partial^{2}\left(P^{2}\right)}{\partial z^{2}}}_{A_{z}}=\phi \frac{\partial P}{\partial t} .
$$

In order to compare the magnitude of the terms $A_{x}$ and $A_{z}$ in the above expression the dimensionless number

$$
\Omega_{x / z}=\frac{K_{x}}{K_{z}} \frac{h_{s}^{2}}{L^{2}} \sim \frac{A_{x}}{A_{z}}
$$

is defined where $L$ is the characteristic length of the skin (in the $X$ direction) and $h_{s}$ its thickness (in the $Z$ direction). A similar number, called aspect ratio, was presented by Šimáček and Advani [27] and quantifies the ratio between in-plane and transverse flow during liquid composite moulding processes. Considering a typical industrial case where $h_{s}=2 \mathrm{~mm}, L=2 \mathrm{~m}$, a transverse permeability of $K_{z}=5 \times 10^{-17} \mathrm{~m}^{2}[9]$ and an in-plane permeability of $K_{x}=6 \times 10^{-14} \mathrm{~m}^{2}[28]$, the aspect ration (Eq. 9) is:

$$
\Omega_{x / z}=1.2 \times 10^{-3} .
$$


In light of the fact that $A_{x} \ll A_{z}$, the through-thickness air flow (term $A_{z}$ in Eq. (8)) is significantly faster than the in-plane flow (term $A_{x}$ ). In this study, a quasi static case will be considered, where the through-thickness air flow always reaches steady-state. Thus, at each time interval, the pressure is equal in the core and through the skin thickness, for each position $(X, Y)$ in the panel.

\subsubsection{Equivalent Permeability}

As suggested above, the pressure through the thickness of the sandwich panel is considered to be homogeneous. Therefore, the focus is on the air flow in the plane of the sandwich. With this aim a Darcy flow

$$
\frac{K_{e q}}{\mu} \nabla_{(x, y)} P(x, y)=-v_{(x, y)}
$$

is retained where $v_{(x, y)}$ is the in-plane apparent velocity of air, $\nabla_{(x, y)}$ is the two-dimensional in-plane spatial derivative operator, and $K_{e q}$ is the in-plane equivalent permeability of the sandwich structure. Note that for the sake of clarity the permeability $K_{e q}$ is scalar, thus considering an isotropic in-plane permeability of the honeycomb structure. The extension to a non-isotropic fabric using a tensorial permeability could be implemented for hybrid skins where $K_{x} \neq K_{y}$, such as in the case of unidirectional prepregs [5] or hybrid skins using both unidirectional and woven fabric.

In order to determine the in-plane equivalent permeability of the sandwich structure, the mesoscopic scale of one honeycomb unit cell was considered. The permeability of the skin contributes to the permeability of the structure, but the air also flows within the honeycomb cell, as illustrated in Fig. 1. As a result, an equivalent in-plane permeability of the honeycomb sandwich is higher than that of the monolithic laminate. As a first approximation, a correction factor $\alpha$ was used, such that

$$
K_{e q}=(1+\alpha) \times K_{x}
$$

where $\alpha$ is positive, and $K_{x}$ is the in-plane permeability of the prepreg skin. In order to quantify this correction factor, a model at the mesoscopic scale was performed. It is detailed in Appendix A and gave a correction factor of

$$
\alpha=0.34 \text {. }
$$

\subsubsection{Flow equation}

In this section, using the behaviour described by Eq. (11) and the conservation of mass, the constitutive equation for the sandwich structure was derived. For the sake of clarity, the study is restricted to a two dimensional problem through-thickness $(Z)$ and in-plane $(X)$. A slice of dimension $d x$ of the sandwich panel, such as the one shown in Fig. 2, was considered. For symmetry, only half of the core was considered. In the skin, Darcy's law reduces to its $x$ component:

$$
\frac{(1+\alpha) K_{x}}{\mu} \frac{\partial P}{\partial x}=v_{x}
$$


Assuming a non-perforated core is used, and the individual cells in the core are isolated, no flow occurs through the cell walls. The upper boundary was sealed due to the use of non-perforated release film. Therefore, flow in the slice only occurs at the skin boundaries, as shown in Fig. 2. The conservation of mass in the slice becomes:

$$
\underbrace{h_{s} v_{x}(x) \rho_{a}(x)}_{\text {in }}-\underbrace{h_{s} v_{x}(x+d x) \rho_{a}(x+d x)}_{\text {out }}=\frac{\partial M}{\partial t},
$$

where $M$ is the total mass of air in the slice. The first term is the inward air flow across the left boundary, the second is the outward flow across the right boundary. The amount of air in the slice $M$ is the sum of air in the skin $M_{s}$ and in the core $M_{c}$

$$
M=M_{s}+M_{c}
$$

Using the volume available in the skin $\phi h_{s} d x$ and in the core $\frac{h_{c}}{2} d x$, Eq. (16) becomes:

$$
M=\rho_{a} \phi h_{s} d x+\rho_{a} \frac{h_{c}}{2} d x
$$

Substituting Eq (15), and dividing by $h_{s} d x$ gives:

$$
\begin{array}{r}
\frac{v_{x}(x) \rho_{a}(x)-v_{x}(x+d x) \rho_{a}(x+d x)}{d x} \\
=\frac{\partial \rho_{a} \times\left(\phi+\frac{h_{c}}{2 h_{s}}\right)}{\partial t} .
\end{array}
$$

For an infinitesimal $d x$, the continuity equation is obtained:

$$
-\frac{\partial\left(\rho_{a} v_{x}\right)}{\partial x}=\frac{\partial \rho_{a}}{\partial t} \times\left(\phi+\frac{h_{c}}{2 h_{s}}\right) .
$$

Considering air as an ideal gas under isothermal conditions, air density $\rho_{a}$ is proportional to pressure $P$, and the continuity equation becomes, in terms of pressure:

$$
-\frac{\partial\left(P v_{x}\right)}{\partial x}=\frac{\partial P}{\partial t} \times\left(\phi+\frac{h_{c}}{2 h_{s}}\right) .
$$

Combined with Darcy's law, the pressure distribution is described by a nonlinear partial differential equation in space and time:

$$
\left(\phi+\frac{h_{c}}{2 h_{s}}\right) \times \frac{\partial P}{\partial t}=\frac{\partial\left(P \frac{(1+\alpha) K_{x}}{\mu} \frac{\partial P(x)}{\partial x}\right)}{\partial x} .
$$

In the case of a three dimensional problem, the same method applied on an infinitesimal column leads to the constitutive equation in the $(X, Y)$ plane:

$$
\left(\phi+\frac{h_{c}}{2 h_{s}}\right) \times \frac{\partial P}{\partial t}=\nabla_{(x, y)} \cdot\left(P \frac{(1+\alpha) K_{x}}{\mu} \nabla_{(x, y)} P\right) .
$$

The mass term, $\phi+h_{c} /\left(2 h_{s}\right)$, shows that the total amount of air to be extracted is the sum of the air contained in the porosity of the skin and the core cells. In the case of a thicker core, the mass term increases, 
showing that a higher amount of air will take longer to extract. In the extreme case where no core is used, the correction factor $\alpha$ is 0 , and the classical air extraction model $[11,14,19,20,23]$ is recovered:

$$
\phi \frac{\partial P}{\partial t}=\nabla_{(x, y)}\left(P \frac{K_{x}}{\mu} \nabla_{(x, y)} P\right) .
$$

\section{2. $1 D$ Analysis}

In this section the one dimension Eq. (21) is solved. This basic case is useful for simple geometries, that reduce to a $1 \mathrm{D}$ problem because of symmetry. It will be shown later, in section 4 , that the $1 \mathrm{D}$ solution can also approximate vacuum hold times for more complex cases.

\subsubsection{Dimensional analysis}

Considering the one dimensional case of a sandwich structure of length $L$, the pressure field, $P(x, t)$, depends on time and space and is governed by Eq. (21). The dimensionless pressure

$$
P^{*}=\frac{P}{P_{\text {atm }}}
$$

was defined using the atmospheric pressure $P_{a t m}$ and the dimensionless position

$$
x^{*}=\frac{x}{L}
$$

using the domain length $L$. Considering uniform material properties $\left(h_{c}, \phi, K_{e q}, \mu\right)$ over the length $L$ in Eq. (21), the in-plane extraction characteristic time

$$
\tau_{x}=\frac{L^{2} \mu\left(\phi+\frac{h_{c}}{2 h_{s}}\right)}{(1+\alpha) K_{x} P_{a t m}}
$$

naturally appears. This characteristic time depends on the process material and geometry, and governs the air extraction duration. Note that in the case of a laminate with no core $\left(h_{c}=0\right)$, the characteristic time introduced by Cender et al. [19], is recovered. A way to obtain this characteristic time will be presented in section 4. Defining the dimensionless time

$$
t^{*}=\frac{t}{\tau_{x}},
$$

equation (21) reduces to its dimensionless form:

$$
\frac{\partial P^{*}}{\partial t^{*}}=\frac{\partial\left(P^{*} \frac{\partial P^{*}}{\partial x^{*}}\right)}{\partial x^{*}} .
$$

\subsubsection{Domain and boundary conditions}

The initial pressure within the honeycomb panel is uniformly atmospheric pressure $P_{a t m}$. One boundary is sealed and vacuum pressure $P_{v}$ is imposed on the other. In terms of dimensionless variables, these initial 
and boundary conditions are:

$$
\begin{aligned}
P^{*}\left(x^{*}, t^{*}=0\right) & =1 \\
\frac{\partial P^{*}\left(x^{*}=1, t^{*}\right)}{\partial x^{*}} & =0 \\
P^{*}\left(x^{*}=0, t^{*}\right) & =\frac{P_{v}}{P_{a t m}}
\end{aligned}
$$

\subsubsection{Resolution}

The boundary value problem governed by Eqs. (28), (29), (30) and (31) was solved using a finite element method in COMSOL Multiphysics. The coordinate $x^{*}$ ranges from 0 to 1 , and $t^{*}$ from 0 to 20 .

This dimensionless solution can be used to predict the pressure versus time for any combination of material properties and domain length using the characteristic time given in Eq. (26).

\subsection{General case: two dimensional resolution}

When dealing with complex geometries, core or skin thickness changes, or several edge breathing strategies, the above one dimensional analysis will lack accuracy. In this section, a framework for solving the general two dimensional constitutive Eq. (22) is presented. The aim is to predict the air extraction in the sandwich panel processed in section 3 .

\subsubsection{Geometry and boundary conditions}

Using symmetry, only half of the domain was considered, as shown in Fig. 3. The core thickness $h_{c}$ depends on the position: from a constant thickness of $2 \mathrm{~cm}$ in the middle of the sandwich, it reduces in the chamfer and reaches 0 in the edge band, according to the experimental setup.

The right, upper and lower boundaries were sealed, resulting in the Neumann boundary condition:

$$
\boldsymbol{v} . \boldsymbol{n}=0,
$$

where $\boldsymbol{n}$ is the outward normal. Using Eq. (11) and (32) the boundary condition in terms of pressure becomes:

$$
\left(\frac{K_{e q}}{\mu} \nabla P\right) \cdot \boldsymbol{n}=0 .
$$

The vacuum pressure was applied along the air evacuation edge, resulting in the Dirichlet boundary condition at the left boundary

$$
P=P_{v}(t)
$$

$P_{v}(t)$ was ramped from atmospheric pressure at time $t=0$ to $P_{0}=5 \times 10^{3} \mathrm{~Pa}$ in $10 \mathrm{~s}$, approximating the experimental procedure.

The initial pressure within the domain was assumed to be atmospheric pressure:

$$
P(t=0)=P_{a t m} .
$$




\subsubsection{Implementation and resolution}

The nonlinear partial differential Eq. (22) was solved numerically in the domain. A finite element method was used in COMSOL to solve for the scalar unknown pressure $P(x, y, t)$. A coefficient form of the PDE module was used. A quadratic interpolation allows for space discretization. The built-in backward Euler method allows for time integration. The non-linearity of Eq. (22) was handled using the built-in modified Newton-Raphson non-linear solver.

The domain shown in Fig. 3 was meshed with 2939 triangular unstructured elements with a refinement at the sandwich/edge-band interface. The parameters used in the simulation are given in Table 1. The solution was computed in roughly 5 seconds using a standard desktop computer.

\section{Experimental validation}

\subsection{Procedure}

A honeycomb panel was manufactured with a $30 \mathrm{~cm} \times 90 \mathrm{~cm}$ honeycomb core and a $7 \mathrm{~cm}$ edge band. The edge band is the section where the upper and lower skins merge, as shown in Fig. 4. The total panel dimensions were $44 \mathrm{~cm} \times 104 \mathrm{~cm}$. The core was cut with a $45^{\circ}$ chamfer using a band saw with a tilting table. Four pressure sensors were inserted in the panel at the locations shown in Fig. 3. The bag-side and tool-side skins were comprised of four plies of plain weave prepreg with a lay-up of $\left[0_{4}\right]$, where $0^{\circ}$ was parallel to the roll direction. The honeycomb core ribbon direction was oriented parallel to the $0^{\circ}$ direction.

\subsubsection{Material}

A plain weave out-of-autoclave $(\mathrm{OoA})$ prepreg material was used in this study. The prepreg was impregnated with Cycom ${ }^{\circledR} 5320$ by Cytec Engineered Materials Inc. The reinforcement fabric had a nominal areal weight of $196 \mathrm{~g} . \mathrm{m}^{-2}$ and an initial resin content of $36 \%$ by weight. The honeycomb core was Aluminum, $20 \mathrm{~mm}$ thick, with $3.1 \mathrm{~mm}$ cell diameter, and a density of $96 \mathrm{~kg} . \mathrm{m}^{-3}$. A structural film adhesive from 3M, AF $163-2 \mathrm{~K}$ with a $294 \mathrm{~g} . \mathrm{m}^{-2}$ weight, was used to co-bond the skin to the core. A surfacing film from Cytec, SM 905 with a $171 \mathrm{~g} . \mathrm{m}^{-2}$ weight was used to minimize surface pitting on the tool-side skin. The consumable materials were fluoronated ethylene propylene (FEP) release film (non-perforated and perforated with $0.38 \mathrm{~mm}$ perforations staggered by $6.35 \mathrm{~mm}$ ), breather, vacuum bag, and sealant tape.

In-plane air permeability measurements were previously reported by Hsiao [28] for Cycom 5320 plain weave prepreg and were used in this study. The air permeability measurements were performed using a vacuum bag set-up where a laminate was wrapped in sealant tape to isolate flow in-plane [28]. Air flow through the laminate was measured for five to ten minutes when vacuum was applied to one side of the laminate as the other side was open to atmosphere. The in-plane permeability results of testing three four-ply laminates are shown in Fig. 5 as a function of debulking time [28]. 


\subsubsection{Sensors}

A patented miniature sensor technology from Convergent Manufacturing Technologies was used to measure the pressure inside the honeycomb core [18]. A piezoresistive silicon micromachined pressure sensor from Measurement Specialties (model number: MS5407-AM) was used [29].

The sensor has a Wheatstone bridge wiring configuration, and therefore requires 4 wires to be soldered to the back side of the carrier. Enamel coated copper magnet wire was used to connect the sensor to the data acquisition system to avoid introducing air leaks along the wire, into the honeycomb panel or vacuum bag. Twenty-eight gauge wire was found to offer the best balance of handling, durability and size; thirty-two and thirty-six gauge wires were evaluated, but those sizes readily became entangled and broke at the solder joints, even with very careful handling.

These pressure sensors offer temperature compensation by embedding a fluorocarbon polymer coated thirty-gauge K-type thermocouple beside the pressure sensor within the honeycomb. The temperature compensation required a four-point calibration at two temperatures: $22{ }^{\circ} \mathrm{C}$ and $125^{\circ} \mathrm{C}$, and two pressures: 0 and $10^{5} \mathrm{~Pa}$. The voltage reading of the sensor was determined at the four points, and a linear response was assumed between the upper and lower bounds of the calibration. This assumption was deemed acceptable in light of the $0.2 \%$ linearity reported by the manufacturer [30].

\subsubsection{Embedding sensors in a honeycomb core}

A simple solution was sought to embed the pressure sensor and pass the wires through the panel without introducing a leak path, and subsequently disrupting the pressure behavior. The pressure sensor did not fit into a single $3.1 \mathrm{~mm}$ honeycomb core cell, therefore a $12 \mathrm{~mm}$ diameter blind hole was drilled $8 \mathrm{~mm}$ deep into the honeycomb core. The sensor was embedded in the core is shown in Fig. 6. Six wires (four for the Wheatstone bridge and two for the thermocouple) were placed in-between two layers of adhesive film, between the pressure sensor and the edge of the core. A high-temperature Viton ${ }^{\circledR}$ fluoroelastomer tubing slice (resistant up to $200{ }^{\circ} \mathrm{C}$ ) was inserted over the metal cap. It prevented incidental contact between the honeycomb cells and the sensor, and between the solder joints (on the bottom of the ceramic carrier) and the composite or adhesive. High-temperature tape was used to secure the two pieces of tubing together; the tape did not cover the sensor.

\subsubsection{Lay-up}

The lay-up of the panel was performed on a $60 \mathrm{~cm} \times 120 \mathrm{~cm}$ aluminum tool plate that was $6 \mathrm{~mm}$ thick. Sealant tape was placed around the perimeter of the tool. A non-perforated release film was placed onto the exposed tool surface, and Flashbreaker ${ }^{\circledR}$ II tape was used to secure the release film to the tool. The surfacing film was placed onto the release film, followed by the first prepreg ply. Edge breathing (fibreglass cloth wrapped around sealant tape) was placed flush against the air evacuation edge (see Fig. 6), and then used as the locating point for the following three tool-side plies. This ensured each prepreg ply was connected 
to the vacuum pump through the edge breathing. After all four layers were laid onto the tool, a layer of perforated release film was placed on top of the skin with one layer of breather, and a loose vacuum bag (to accommodate the core in future lay-up steps). The entire assembly was debulked for $1 \mathrm{~h}$ with $10^{4} \mathrm{~Pa}$ of vacuum under the bag.

During the tool-side skin debulking, the sensors were installed in the core as described above. After the debulking bag was removed from the tool, the completed assembly, consisting of the core with the embedded sensors fully covered with adhesive, was carefully placed onto the debulked tool-side skin. To prevent leaks, the sensors ran parallel to the edge breathing, were spaced $1 \mathrm{~cm}$ apart, and sandwiched between two layers of sealant tape where exiting the panel and vacuum bag. After the core was located, the bag-side adhesive was placed over the core, followed by the first prepreg ply. The entire assembly was debulked for $10 \mathrm{~min}$. This procedure was repeated for the three remaining bag-side plies. Sealant tape was placed flush around the remaining three edges to prevent air flow during the vacuum hold and cure.

Draping the bag-side prepreg plies over the chamfered core required stretching the prepreg by 4 to $5 \mathrm{~mm}$ to avoid wrinkles. This introduced some fibre waviness, as shown in Fig. 4, however, this also created an overlap at the edge breathing. The stretched prepreg was trimmed with scissors to ensure a flush fit with the edge breathing.

New sealant tape and a new vacuum bag were installed prior to the lengthy vacuum hold. Two vacuum ports were used. One was connected to the vacuum pump, the other was connected to a pressure transducer (range: 0 to $10^{5} \mathrm{~Pa}$ ) to record the vacuum bag pressure using the same data acquisition system as the embedded sensors.

\subsection{Validation}

The honeycomb panel was held under vacuum for $18 \mathrm{~h}$ at room temperature. The pressure measured at the four sensors are shown in Fig. 7. The difference between the model predictions and experimental measurements is likely due to micro-structure of the prepreg skin and its permeability.

In Fig. 7, a constant non-debulked permeability:

$$
K_{x}=1.2 \times 10^{-13} \mathrm{~m}^{2},
$$

was applied from Fig. 5, using the maximum bound, as illustrated by the dashed line. Since the plies were debulked for 10 minutes during lay-up, it is thought that the cells are not well sealed to the skin, and resulting in a high under prediction of air extraction (under prediction of the correction factor $\alpha$ described in section Appendix A). The square root of the mean squared pressure error between the experimental measurements and the prediction is $0.36 \times 10^{5} \mathrm{~Pa}$.

In order to identify if the consolidation of the prepreg during the vacuum hold reduced the air permeability, the vacuum was released, allowing atmospheric pressure to re-entre the honeycomb panel, which 
required $9 \mathrm{~h}$ to reach equilibrium. The debulked air permeability values in Fig. 5 were used to predict the increase in pressure and are compared with the sensor measurements in Fig. 8.

After the skins were fully debulked, material properties reported in Table 1 are representative. Using these values, the two-dimensional model (described in section 2.3) was adapted (using an initial vacuum pressure in the part and an atmospheric boundary condition at the breathing edge). The pressure predictions (shown in Fig. 8) accurately matched the sensor measurements. The square root of the mean squared pressure error is now $4.8 \times 10^{3} \mathrm{~Pa}$, offering a much stronger correlation after the panel was thoroughly debulked.

A second vacuum hold of $16 \mathrm{~h}$ was performed and the measured pressure response is compared to model predictions in Fig. 9. Agreement between the predicted and measured pressure are very close, confirming the accuracy of the proposed model when applied to a well debulked panel. The square root of the mean squared pressure error is below $2.5 \times 10^{3} \mathrm{~Pa}$. The difference between the measured pressure responses in Fig. 7 and Fig. 9 clearly show the effect of debulking during lay-up on the duration of the vacuum hold needed to evacuate entrapped air prior to cure.

Debulking during lay-up may reduce ply consolidation defects during cure, such as ply bridging, but will require longer vacuum hold times to extract air and volatiles that lead to interlaminar voids during cure. The effect of debulking can be incorporated using this model with an adjustable correction factor $\alpha$ to help develop robust process specifications.

\section{Application}

In this section, the model was used to predict the pressure drop during a vacuum hold for industrial structures. Of interest, is the position where the maximum pressure occurs in the part. This is likely to be at the furthermost point from the edge breathing. The model was used to predict the minimum achievable pressure versus vacuum hold time for different industrial cases.

\subsection{Vacuum hold time}

The one dimensional model presented in section 2.2 was used to create a design chart as shown in Fig. 10.

\subsubsection{Construction}

For a given core to skin thickness ratio $h_{c} / h_{s}$ and a given domain length $L$, Eq. (26) gives the characteristic time $\tau$. Using the material data for Cycom 5320 (Table 1), the isovalues of $L$ are plotted on a $\left(\tau, h_{c} / h_{s}\right)$ map in the middle graph of Fig. 10. For a more general case, where $K_{x}$ would differ from the value given in Table 1 , the isovalues of $L / K_{x}^{2}$ are plotted on the same coordinate system on the top graph of Fig. 10 .

For a given characteristic time $\tau$ and a given vacuum hold time $t$, the dimensionless time $t^{*}$ was obtained. Using the dimensionless solution, the pressure loss at the furthermost position from the edge breathing was directly obtained. The isovalues of that pressure are plotted on a $(\tau, t)$ map on the bottom graph. 


\subsubsection{Example of use}

For a given geometry $h_{c}, h_{s}$ and $L$, one point is obtained on either the middle chart for Cycom 5320 or the top chart for a material with a different in-plane permeability. Drawing a vertical line down to the bottom chart gives the vacuum hold times needed to achieve a maximum pressure in the part.

As a first example, prediction for the lab scale experiment detailed in section 3 was obtained. In the geometry (Fig. 3), the edge band was wide compared to the sandwich width (about one third). An equivalent core thickness $\tilde{h}_{c}$ is simply defined as the average core thickness in the cross section:

$$
\tilde{h}_{c}=\frac{w \times h_{c}}{w+2 \times e_{b}}
$$

The parameters in Table 1 give:

$$
\frac{\tilde{h}_{c}}{h_{s}}=13.8 .
$$

Subsequently, for a length $L=0.9 \mathrm{~m}$, the characteristic time obtained on the middle graph of Fig. 10 is

$$
\tau \sim 1.35 \times 10^{4} \mathrm{~s}
$$

which corresponds to $13.5 \mathrm{~h}$ to reach $2 \times 10^{4} \mathrm{~Pa}$ in the lower graph. This is in close agreement with the pressure measured by the furthermost sensor (number 4) on Fig. 9.

\subsection{Case study: large scale structures}

In this section, a representative airplane structure was studied. A generic fuselage barrel geometry is shown in Fig. 11. A series of windows and an exit door are on both sides of the cylinder, and one cargo door is positioned on the lower half of the part. Two processing strategies were compared:

1. Full-barrel processing: the full cylinder is manufactured in one shot. The edge breathing was located on the two extreme perimeters of the cylinder.

2. Third-barrel processing: the cylinder is manufactured in three distinct parts that will be assembled in a second step. On each third of cylinder the edge breathing was positioned around the whole perimeter of the panel.

The industrial sandwich properties are given in Table 2, which is different from the lab scale experiment presented above. For each strategy, the full two dimensional simulation was run on an "unfolded" geometry. The most complex third (containing all doors and windows) was considered in the third-barrel strategy.

\subsubsection{Full-barrel strategy}

The predicted pressure field in the unfolded full barrel structure after a $60 \mathrm{~h}$ vacuum hold is presented in Fig. 12. As expected, the maximum pressure was located in the central section of the barrel. It reached a minimum of $0.79 \times 10^{5} \mathrm{~Pa}$ after $60 \mathrm{~h}$, which may not be acceptable to ensure a sufficient consolidation during processing. 
This result can be approximated using the design graph in Fig. 10. The current core to skin ratio is $h_{c} / h_{s}=10$, and the maximum distance from the edge breathing is half the cylinder length, i.e. $7.5 \mathrm{~m}$, which gives a characteristic time of

$$
\tau=6.8 \times 10^{5} \mathrm{~s}
$$

and a highest pressure

$$
P_{\max }=0.8 \times 10^{5} \mathrm{~Pa}
$$

in the part after $60 \mathrm{~h}$ vacuum hold. This is in close agreement with the two dimensional finite element prediction above, confirming the usefulness of Fig 10 to approximate vacuum hold times in a production environment.

\subsubsection{Third-barrel strategy}

Fig. 13 shows the predicted pressure for the third barrel after $60 \mathrm{~h}$ vacuum hold. As expected, the maximum pressure in the part was located in the centre of the panel. Due to a tortuous air extraction path, the pressure is also higher in the intricate regions, such as behind the doors. This results in regions of higher final pressure. The maximum pressure reduces to $0.58 \times 10^{5} \mathrm{~Pa}$ which is lower than in the full barrel strategy.

This result was approximated using the design graph. The maximum distance to edge breathing is now one half of the panel width, which is a third of the perimeter of the full barrel, i.e. $\pi / 6 \times 10 \mathrm{~m}=5.2 \mathrm{~m}$. The design graph predicts a maximum pressure of $0.61 \times 10^{5} \mathrm{~Pa}$ after 60 hour vacuum hold. This slightly over-predicts the two-dimensional simulated pressure because the one dimensional approach does not take into account the edge effects that can be seen on Fig. 13. However, even with intricate features, the 1D approximation is within $5 \%$ of the $2 \mathrm{D}$ numerical simulation.

\subsection{Transverse vs. in-plane extraction}

\subsubsection{Through-thickness evacuation model}

This section recalls the modelling of the through-thickness evacuation that would happen if a perforated release film was used. In this case, the problem becomes a one dimensional transverse problem only. The pressure differential between the core $P$, and the vacuum bag $P_{v}$ governs the pressure profile in the skin $P_{s}(z)$. Considering a quasi-static case and the ideal gas law, Tavares et al. [14] or Kratz and Hubert [9] Eq. (8) obtained the governing equation

$$
h_{c} \frac{d P}{d t}=-\frac{K_{z}}{\mu} \frac{\left(P^{2}-P_{v}^{2}\right)}{2 h_{s}}
$$

for the core pressure evolution. Using the dimensionless notation defined in section 2.2 Eq. (42) reduces to

$$
\frac{d P^{*}}{d t^{*}}=-\left(P^{* 2}-\left(\frac{P_{v}}{P_{a t m}}\right)^{2}\right)
$$


where the dimensionless time

$$
t^{*}=\frac{t}{\tau_{z}}
$$

is now defined using the through-thickness characteristic time

$$
\tau_{z}=\frac{2 \mu h_{s} h_{c}}{K_{z} P_{a t m}} .
$$

Eq. (43) can be solved numerically using the initial condition

$$
P^{*}\left(t^{*}=0\right)=1 .
$$

\subsubsection{Comparison}

In light of the high core pressure after lengthy $60 \mathrm{~h}$ vacuum holds, a transverse evacuation strategy could be considered to reduce vacuum hold prior to cure. Using the in-plane one dimensional model and the transverse through-thickness evacuation model presented in section 4.3.1, the two extraction methods can be compared. Equating the in-plane and transverse characteristic times (given in Eqs. (26) and (45)) gives a transition characteristic length of

$$
L_{c}=\sqrt{\frac{2(1+\alpha) K_{x} h_{s} h_{c}}{K_{z}\left(\phi+\frac{h_{c}}{2 h_{s}}\right)}} .
$$

In typical industrial cases, $\phi \sim 0.3$ and $h_{c} \gg h_{s}, L_{c}$ can be approximated by

$$
L_{c}=2 h_{s} \sqrt{\frac{(1+\alpha) K_{x}}{K_{z}}}
$$

which is in agreement with the aspect ratio defined by S̆imáček and Advani [27]. For parts longer than $L_{c}$, transverse extraction will be faster than in-plane.

The transverse and in-plane extraction were compared for different lengths. The properties used are that of the non-debulked (optimistic) Cycom 5320 material given in Table 1, and $K_{z}$ given in Table 3 . The correction factor $\alpha$ was increased to 3, in order to account for the non-debulked effect discussed in section 3.2. This provided a better fit to the experimental values reported in Fig. 7. The iso-pressures obtained for the fastest strategy (in-plane or transverse extraction) are plotted in Fig. 14. It shows that for small parts, in-plane extraction is faster. The characteristic length obtained using equation (48) is

$$
L_{c}=0.2 \mathrm{~m}
$$

which appears to be a good evaluation of the transition length in the map. Since $L$ is the distance from the edge breathing, a length of $0.2 \mathrm{~m}$ represents a panel of width $0.4 \mathrm{~m}$, assuming edge breathing is applied on all sides. 


\section{Conclusion}

A model was developed to predict the in-plane air evacuation of honeycomb sandwich panels in out-ofautoclave prepreg manufacturing. The model predicts pressure drop versus vacuum hold time in the three dimensional structure, prior to cure. The model was solved using finite elements methods, and validated with an experimental campaign using a representative $1 \mathrm{~m}$ long panel. The representative panel was instrumented with pressure sensors to measure the pressure gradient within the panel. The model was accurate for a well de-bulked lay-up, and under-predicted the pressure drop for a lay-up with less de-bulking.

The simulation was applied to study industrial geometries. However, general guidelines were derived from the model, and found to closely approximate the necessary vacuum hold for any material and geometrical parameters. For a given material and lay-up, the evacuation time increases in a quadratic manner with panel dimensions. As a result, in-plane gas evacuation of honeycomb panels may be unrealistic in a production environment. For the materials used in this study and parts larger than $0.4 \mathrm{~m}$, air would be more rapidly evacuated using a transverse evacuation strategy.

\section{Acknowledgements}

This work received financial support from the Natural Sciences and Engineering Research Council of Canada (NSERC), the Consortium for Research and Innovation in Aerospace in Quebec (CRIAQ), Bell Helicopter Textron Canada, Bombardier Aerospace, and Delastek. We would like to thank Bell Helicopter Textron Canada and Bombardier Aerospace for providing the materials used in this study. The authors would like to thank Convergent Manufacturing Technologies for granting special permission to use their patented miniature pressure sensor technology in this work.

\section{References}

[1] Kratz J, Hubert P. Processing out-of-autoclave honeycomb structures: Internal core pressure measurements. Composites Part A: Applied Science and Manufacturing 2011;42(8):1060-5.

[2] Schlimbach J, Ogale A. Out-of-autoclave curing process in polymer matrix composites. In: Advani SG, Hsiao KT, editors. Manufacturing techniques for polymer matrix composites (PMCs). Philadelphia, PA: Woodhead Publishing; 2012, p. 435-81.

[3] Cytec . Cycom 5320 epoxy resin system. Technical data sheet. AECM-00017. 2012.

[4] Sharma S, Siginer DA. Permeability Measurement Methods in Porous Media of Fiber Reinforced Composites. Applied Mechanics Reviews 2010;63(2).

[5] Arafath A, Fernlund G, Poursartip A. Gas transport in prepregs: model and permeability experiments. In: ICCM-17. Edinburgh, UK; 2009,.

[6] Louis B, Hsiao K, Fernlund G. Gas permeability measurements of out of autoclave prepreg MTM45-1/CF2426A. In: SAMPE. Seattle, WA, USA; 2010,

[7] Xin C, Li M, Gu Y, Li Y, Zhang Z. Measurement and analysis on in-plane and through-thickness air permeation of fiber/resin prepreg. Journal of Reinforced Plastics and Composites 2011;30(17):1467-79.

[8] Hickey C, Timms J, Bickerton S. Compaction response and air permeability characterization of out-of-autoclave prepreg materials. In: 11th International Conference on Flow Processing in Composite Materials (FPCM-11). Auckland, NZ; $2012,$.

[9] Kratz J, Hubert P. Anisotropic air permeability in out-of-autoclave prepregs: Effect on honeycomb panel evacuation prior to cure. Composites Part A: Applied Science and Manufacturing 2013;49:179-91.

[10] Centea T, Hubert P. Measuring the impregnation of an out-of-autoclave prepreg by micro-CT. Composites Science and Technology 2011;71(5):593-9. 
[11] Advani S, Sozer E. Process modeling in composites manufacturing. Boca Raton, FL: CRC Press; 2011.

[12] Sozer E, Šimáček P, Advani SG. Resin transfer molding (RTM) in polymer matrix composites. In: Advani SG, Hsiao KT, editors. Manufacturing techniques for polymer matrix composites (PMCs). Philadelphia, PA: Woodhead Publishing; 2012, p. 243-309.

[13] Hubert P, Fernlund G, Poursartip A. Autoclave processing for composites. In: Advani SG, Hsiao KT, editors. Manufacturing techniques for polymer matrix composites (PMCs). Philadelphia, PA: Woodhead Publishing; 2012, p. 414-34.

[14] Tavares SS, Michaud V, Månson JA. Through thickness air permeability of prepregs during cure. Composites Part A: Applied Science and Manufacturing 2009;40(10):1587-96.

[15] Tavares SS, Caillet-Bois N, Michaud V, Månson JA. Non-autoclave processing of honeycomb sandwich structures: Skin through thickness air permeability during cure. Composites Part A: Applied Science and Manufacturing 2010;41(5):646-52.

[16] Tavares SS, Caillet-Bois N, Michaud V, Månson JA. Vacuum-bag processing of sandwich structures: Role of honeycomb pressure level on skin-core adhesion and skin quality. Composites Science and Technology 2010;70(5):797-803.

[17] Tavares SS, Michaud V, Månson JA. Assessment of semi-impregnated fabrics in honeycomb sandwich structures. Composites Part A: Applied Science and Manufacturing 2010;41(1):8-15.

[18] Lane M, Poursartip A, Fernlund G, Willden K, Nelson K. Robust processing of composite structures. In: SAMPE Conference and Exhibition. Seattle, WA, USA; 2010,.

[19] Cender TA, Šmáček P, Advani SG. Degassing of a Partially Impregnated Fabric as a Function of Resin Saturation for Out-of-Autoclave Processing. In: SAMPE. Baltimore; 2012,.

[20] Wu Y, Pruess K. Gas flow in porous media with Klinkenberg effects. Transport in Porous Media 1998;32:117-37.

[21] Klinkenberg D. The permeability of porous media to liquids and gases, Drilling and Production Practice. American Petroleum Institute 1941;

[22] Hou Y, Comas-Cardona S, Binetruy C, Drapier S. Gas transport in fibrous media: Application to in-plane permeability measurement using transient flow. Journal of Composite Materials 2012;47(18):2237-47.

[23] Scheidegger AE. The physics of flow through porous media. University ed.; Toronto; 1957.

[24] Muskat M. The Flow of Homogeneous Fluids through Porous Media. J. w. edwa ed.; Ann Arbor, MI; 1946.

[25] Shan C. Analytical Solutions for Determining Vertical Air Permeability in Unsaturated Soils. Water Resource Research $1995 ; 31(9): 2193-200$

[26] Vàzquez JL. The porous medium equation. New York: Oxford Mathematical Monographs; 2007.

[27] Šmáček P, Advani SG. Desirable features in mold filling simulations for Liquid Composite Molding processes. Polymer Composites 2004;25(4):355-67.

[28] Hsiao K. Gas Transport And Water Vapourization in Out-of-autoclave Prepreg Laminates. Master thesis; The University of British Columbia; 2012.

[29] Kratz J, Hubert P. In-situ monitoring of honeycomb pressure using embedded sensors. In: SAMPE 2011 Spring Technical Conference and Exhibition. Long Beach, CA; 2011,.

[30] Intersema Sensoric SA . MS54xx Miniature SMD Pressure Ssensors. Product data sheet $000054 x x 280$ ECN 1243.2009.

\section{Appendix A. Sandwich equivalent permeability}

Even with an isotropic in-plane permeability of the skins, the sandwich structure is anisotropic due to the hexagonal pattern of the honeycomb cells. Thus, in-order to evaluate the value of the equivalent air permeability (Eq. (12)) of the sandwich structure, an analysis was performed on two representative volume elements (RVE), at the scale of the unit cell. Using symmetries and periodicity, the RVEs considered reduced to the ones shown in Fig. 15. The case $0^{\circ}$ is considered to obtain the equivalent permeability $K_{e q}^{0}$ in the $0^{\circ}$ direction, and the case $90^{\circ}$ to obtain the equivalent permeability $K_{e q}^{90}$ in the $90^{\circ}$ direction. Using symmetry through-thickness, one single skin was considered. It is sealed on one face (the bag- or tool-side), and in contact with the air of the honeycomb cell on the other face.

The simple case of a steady state incompressible Darcy's flow was considered in the skin. For a constant fluid viscosity, the constitutive equation classically reduces to:

$$
K_{x} \frac{\partial^{2} P}{\partial x^{2}}+K_{x} \frac{\partial^{2} P}{\partial y^{2}}+K_{z} \frac{\partial^{2} P}{\partial z^{2}}=0
$$

which is a linear partial differential equation. To estimate the equivalent permeability of the RVE, a pressure differential was imposed on the boundary and the air flux transiting from cell to cell was estimated. 


\section{$0^{\circ}$ direction}

The case $0^{\circ}$ representative volume element is shown in Fig. 16. To estimate the equivalent permeability in the $0^{\circ}$ direction, a pressure differential was imposed in this direction in the RVE: $P_{1}$ was imposed at $\Gamma_{L}$ and $\Gamma_{1}$, and $P_{2}$ at $\Gamma_{R}$ and $\Gamma_{2}$. The other boundaries were kept sealed.

Using the superposition principle, this problem was separated in two subproblems:

- $P_{1}$ imposed at $\Gamma_{L}, P_{2}$ at $\Gamma_{R}$ and $\Gamma_{1}$ and $\Gamma_{2}$ were sealed. The problem comes down to a classical 1D Darcy's flow with the permeability $K_{x}$ of the skin

- $P_{1}$ imposed at $\Gamma_{1}, P_{2}$ at $\Gamma_{2}$, and $\Gamma_{L}$ and $\Gamma_{R}$ were sealed. The problem was solved using a finite element method in COMSOL, and the parameters are given in Table 3 . The obtained pressure field is shown in Fig. 17. Using the total flux $q_{1 \rightarrow 2}$ transiting from cell 1 to cell 2 , the equivalent in-plane permeability, $K_{e q}^{0}$, of the system was identified using Darcy's law:

$$
-\frac{K_{e q}^{0}}{\mu} \frac{P_{2}-P_{1}}{l}=q_{1 \rightarrow 2} .
$$

and gave $K_{e q}^{0,2}=0.345 \times K_{x}$.

Summing the two problems gave the equivalent in-plane permeability of the honeycomb sandwich:

$$
K_{e q}^{0}=\left(1+\alpha_{0}\right) K_{x}
$$

with

$$
\alpha_{0}=0.345 \text {. }
$$

\section{$90^{\circ}$ direction}

The case $90^{\circ}$ representative volume element is shown in Fig. 16. Once again, $P_{1}$ was imposed at $\Gamma_{L}$ and $\Gamma_{1}$, and $P_{2}$ at $\Gamma_{R}$ and $\Gamma_{2}$. Because of symmetry, $P_{i}=\left(P_{1}+P_{2}\right) / 2$ was imposed at the intermediate cell $i$. Following the same approach, as for the $0^{\circ}$ case, two pressure fields are added the pressure field obtained numerically with $P_{1}$ imposed on $\Gamma_{1}, P_{2}$ on $\Gamma_{2}$, and $P_{i}$ on $\Gamma_{i}$ is shown on Fig. 18. Summing up the fluxes out of cell 1 and cell i before using Eq. (A.2) gives a value

$$
\alpha_{90}=0.335 .
$$

The two values of $\alpha$ obtained in direction $0^{\circ}$ and $90^{\circ}$ (Eq. (A.4) and (A.5)) are very similar (within 2\%) and show that the hexagonal structure of the honeycomb does not lead to a high anisotropy of the equivalent permeability of the sandwich structure. An isotropic in plane equivalent permeability

$$
K_{e q}=(1+\alpha) K_{x}
$$

is therefore retained for the honeycomb sandwich panel where

$$
\alpha=0.34 .
$$




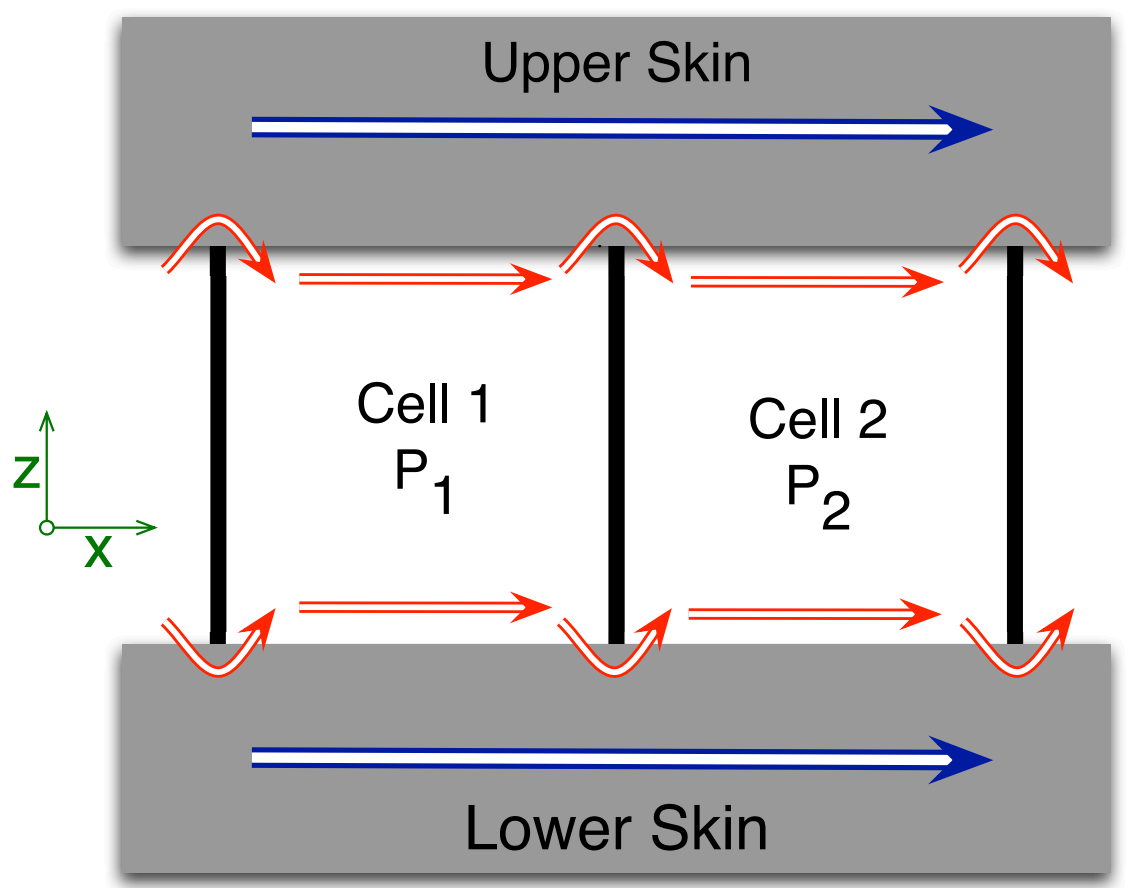

Figure 1: In-plane air-flow phenomena in a honeycomb sandwich structure. The blue arrow represent term 1 in Eq. (12) whereas the red arrows represent the term $\alpha$. 


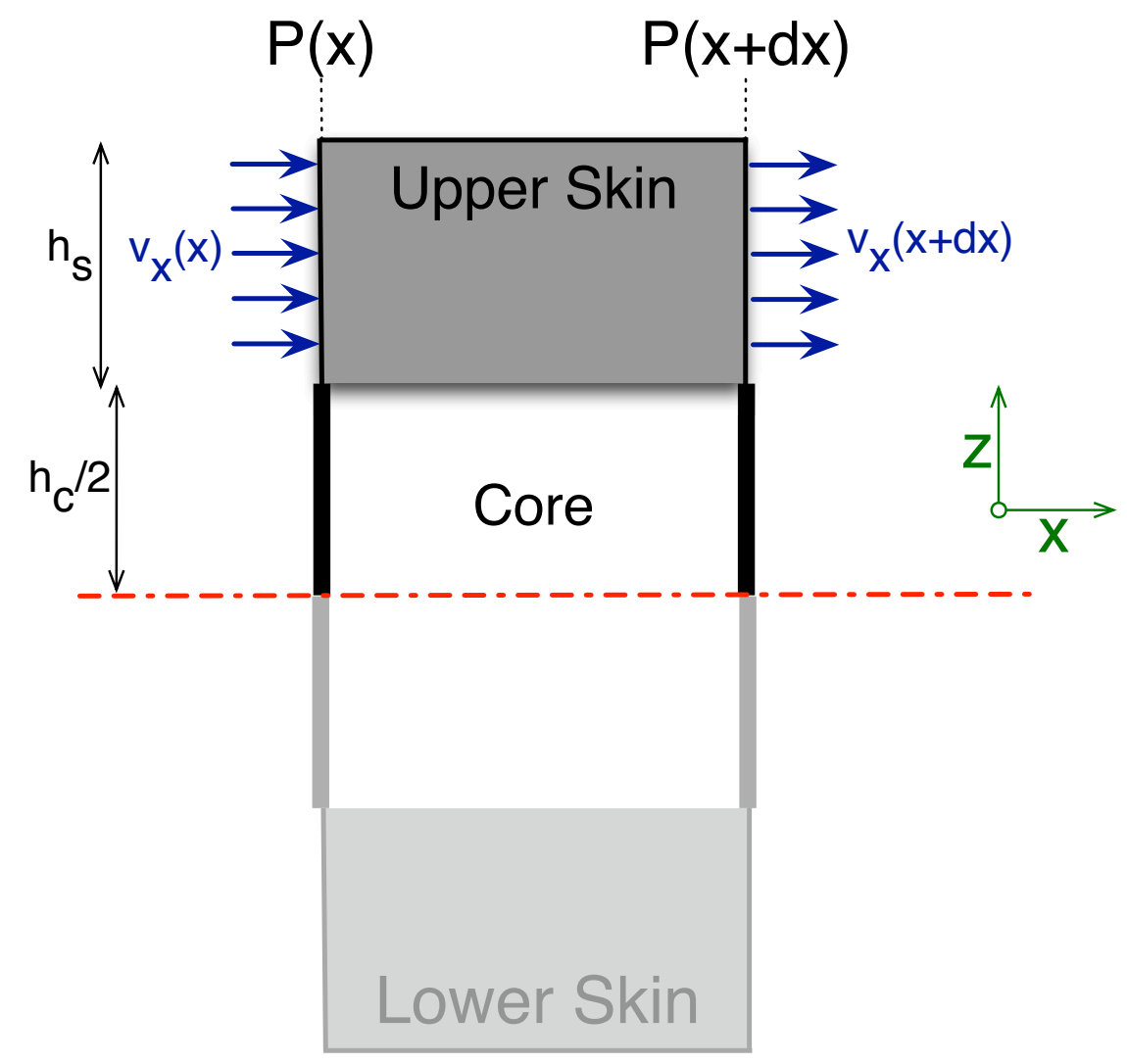

Figure 2: Infinitesimal slice of a honeycomb panel.

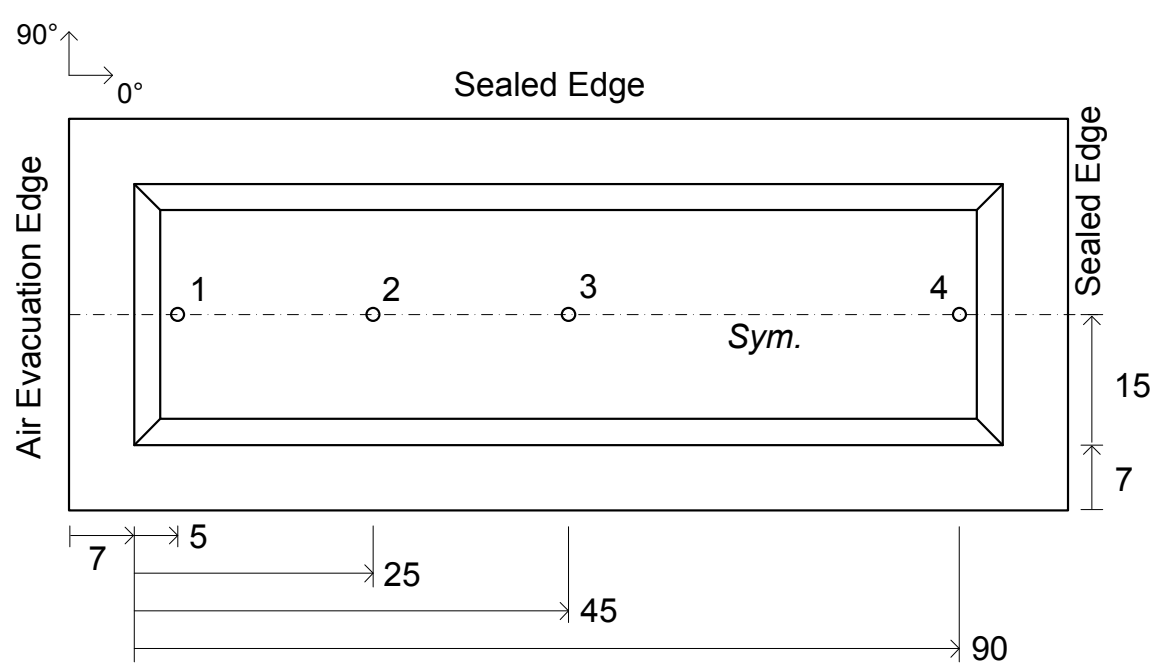

Figure 3: Honeycomb panel dimensions (in $\mathrm{cm}$ ) and the placement of four pressure sensors along the axis of symmetry. 


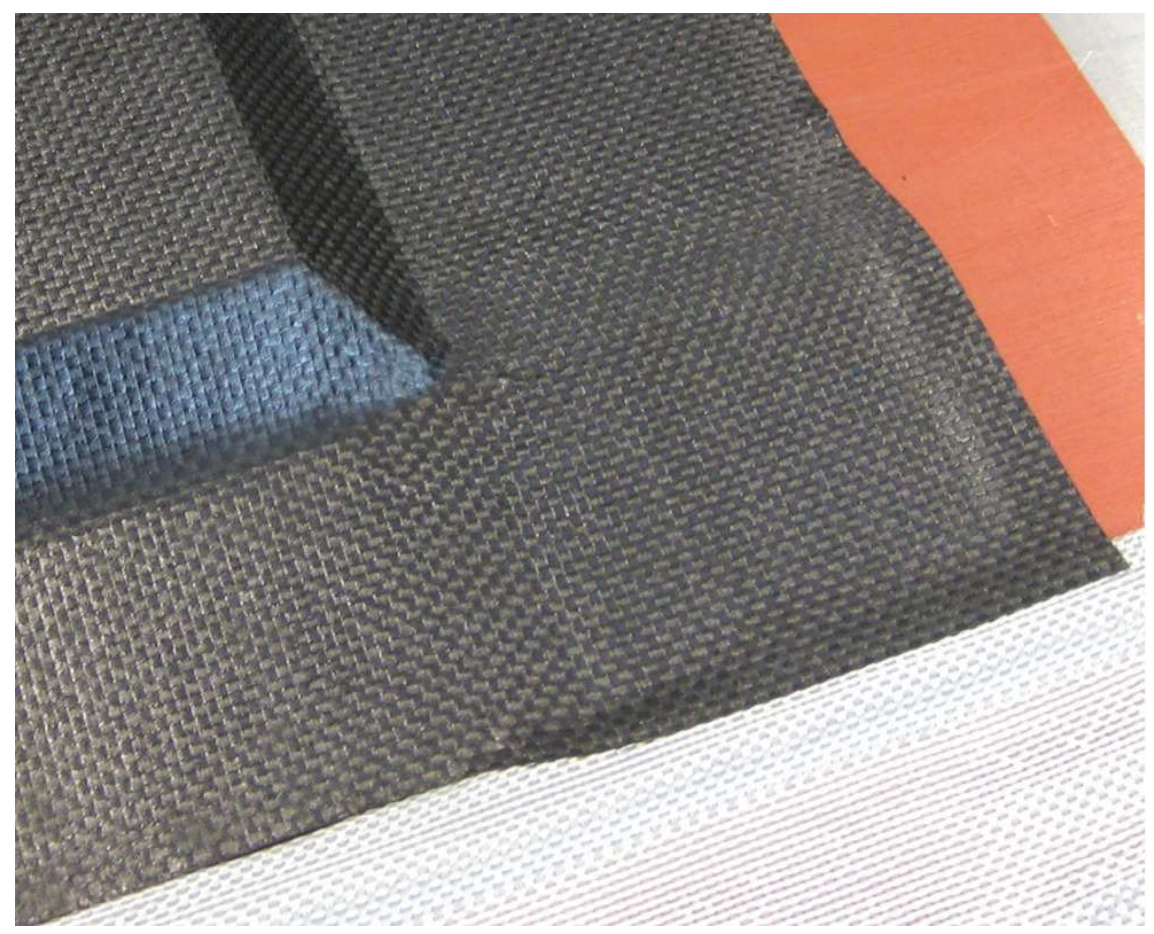

Figure 4: Stretched prepreg at corner before trimming. The two skins merge and overlap on the edge band.

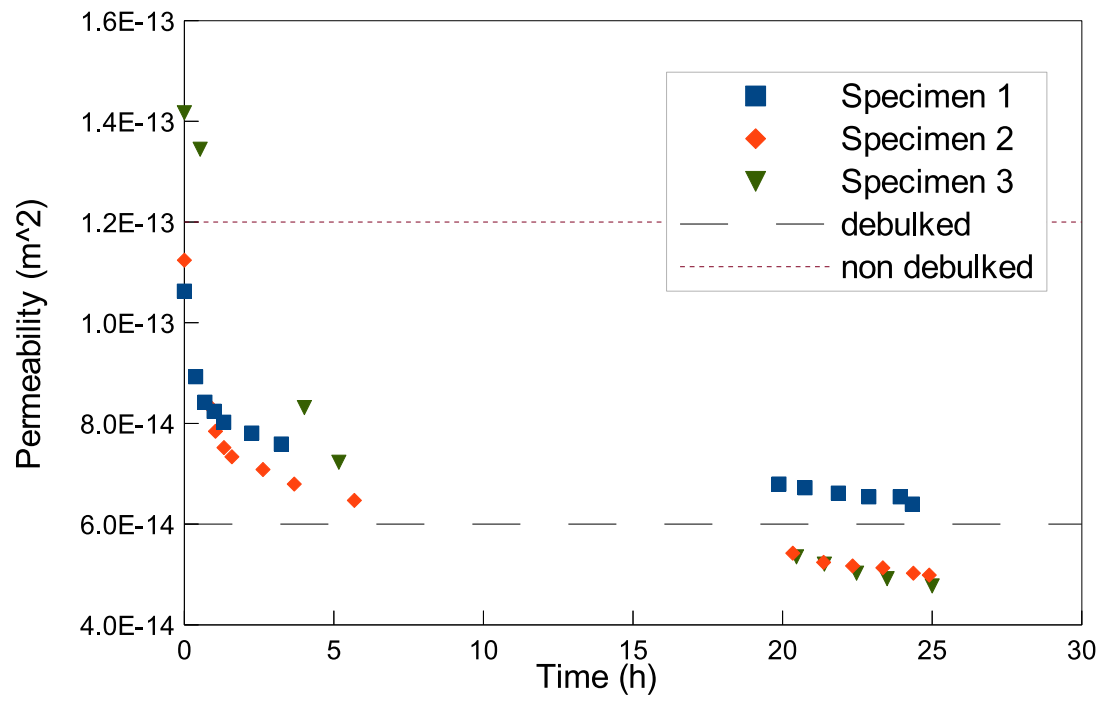

Figure 5: In-plane permeability of Cycom ${ }^{\circledR} 5320$ plain weave prepreg versus debulk time, as measured by Hsiao [28]. Dashed lines represent the debulked and non-debulked permeability values used in this study. 


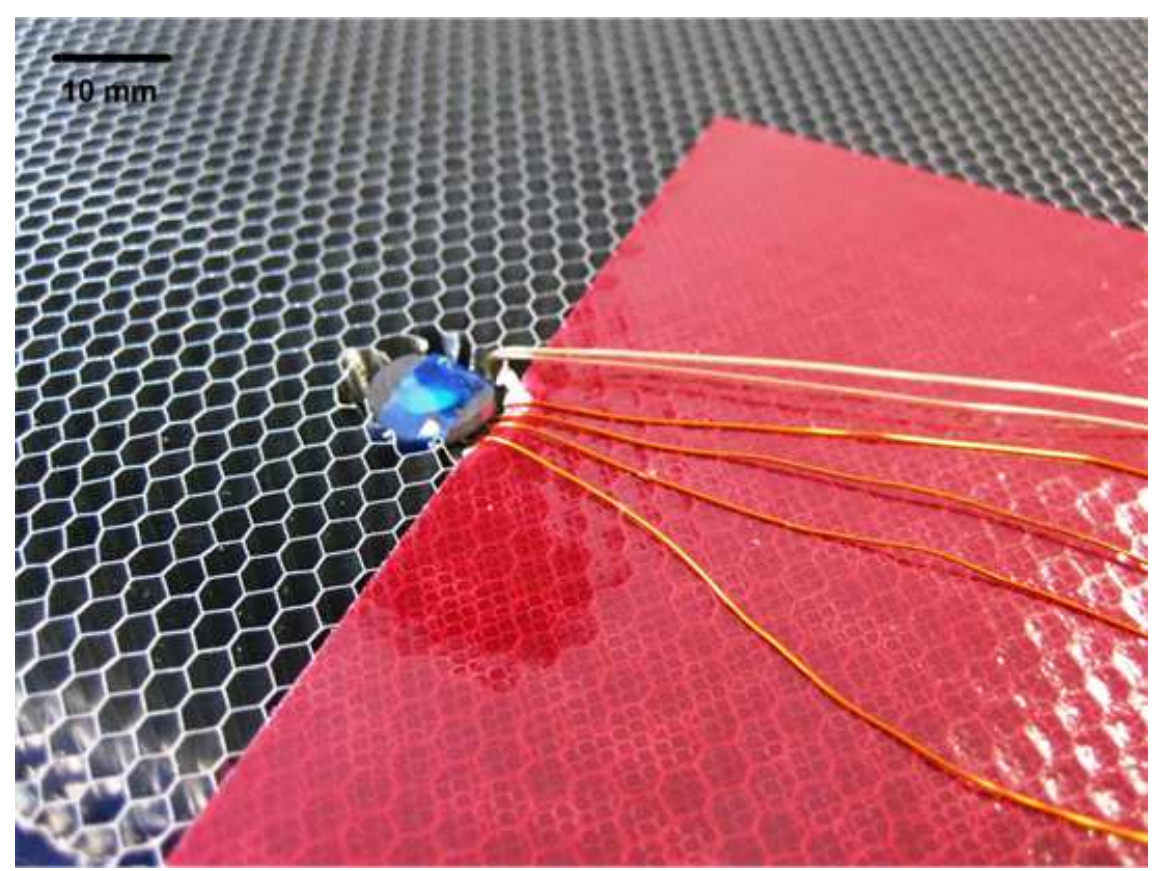

Figure 6: Sensor embedded in honeycomb core, protected by fluoroelastomer tubing. The wires are above one layers of adhesive film. The second layer of adhesive completely covers the honeycomb core.

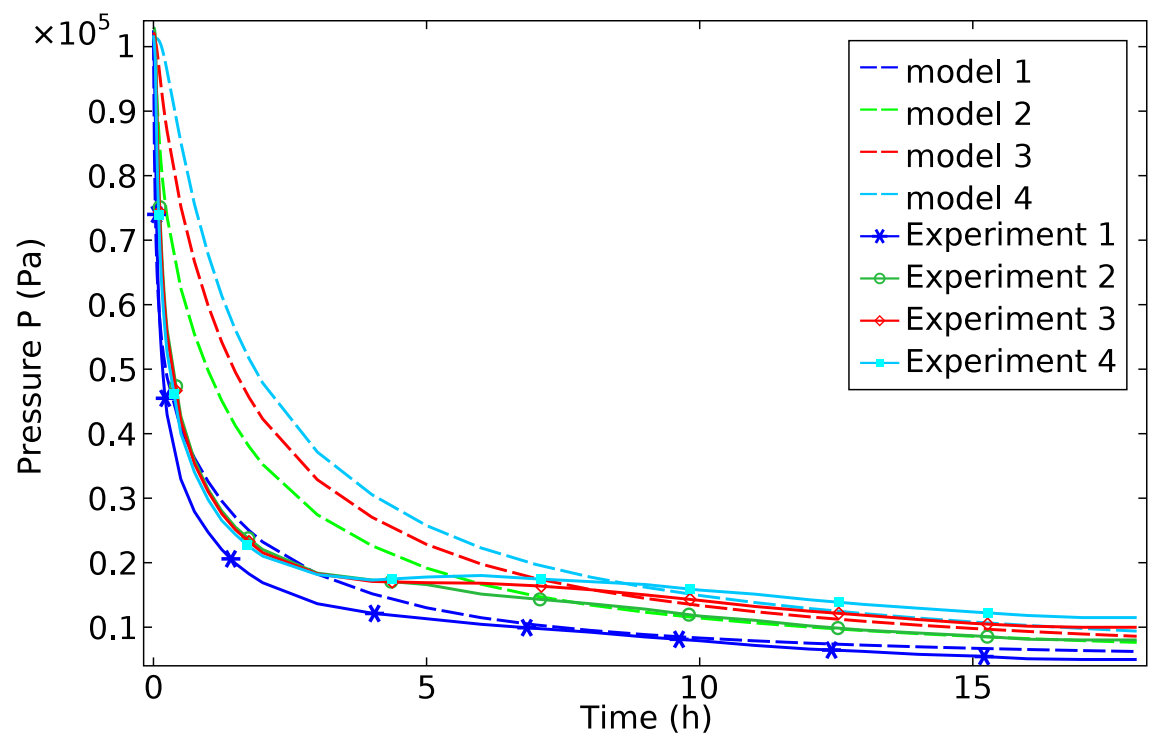

Figure 7: Pressure versus time at sensor locations during the initial vacuum hold. Comparison between prediction (Eq 22) and measurements identify a noticeable discrepancy between locations 2 through 4 . 


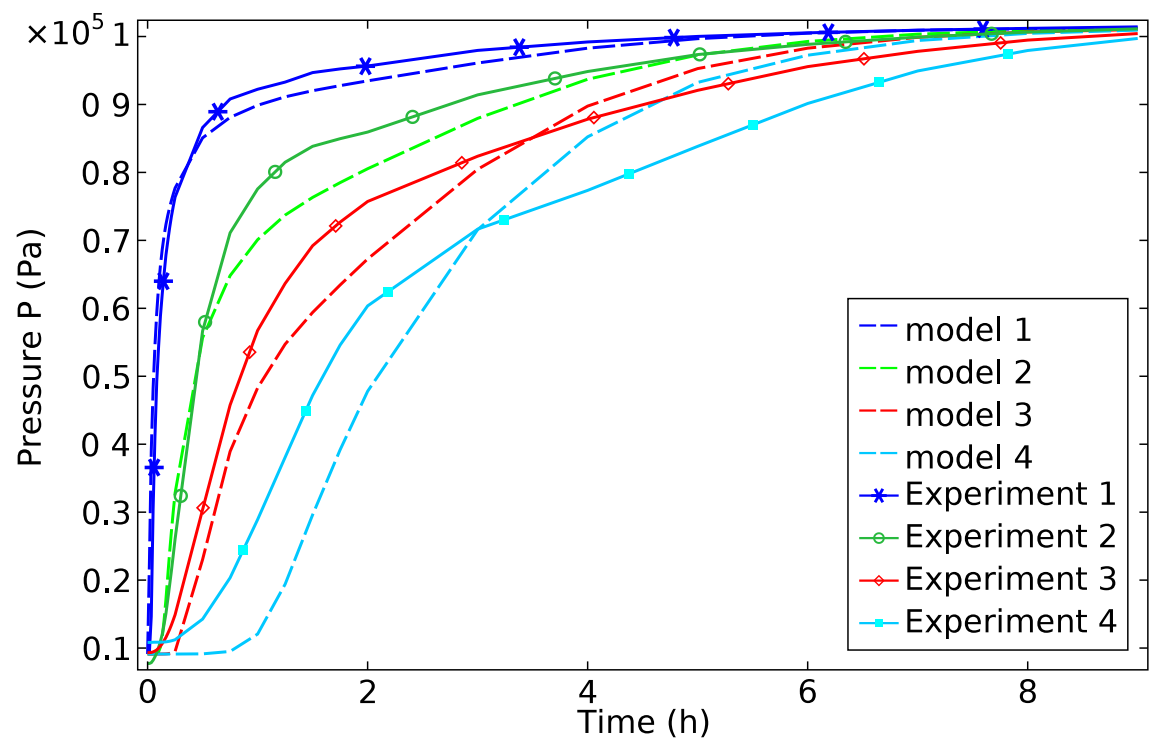

Figure 8: Pressure versus time at the sensor location during the vacuum release. Comparison between predicted and measured pressure correlate after the panel was throughly debulked.

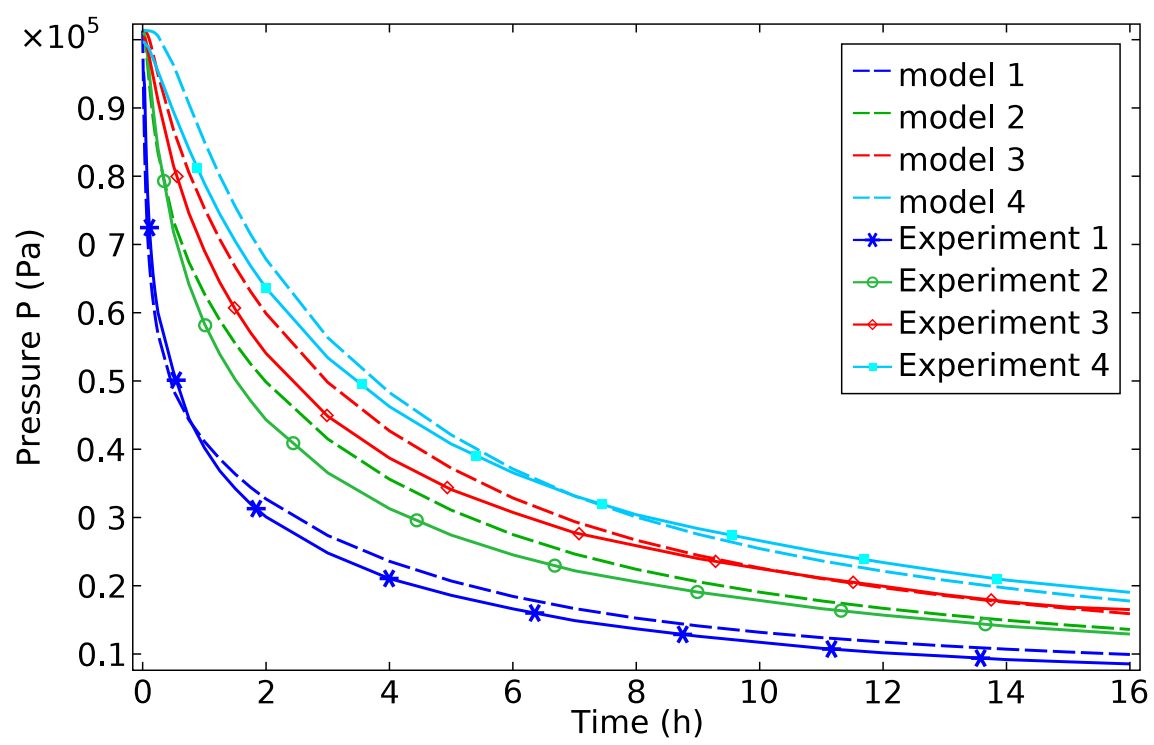

Figure 9: Pressure versus time at the sensor locations during the second vacuum hold. Comparison between predicted and measured pressure are very close. Lengthy debulking will require longer vacuum hold times prior to cure. 

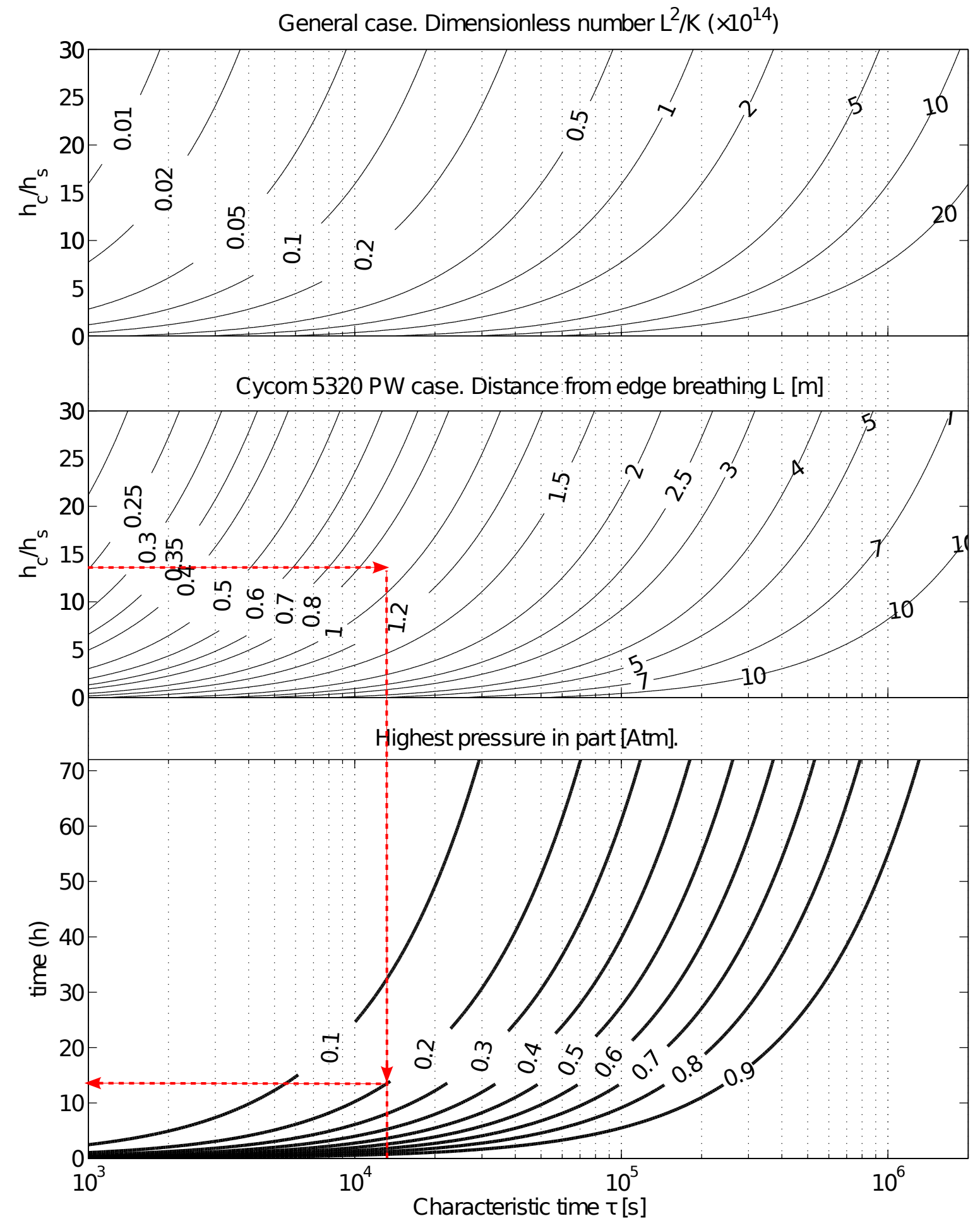

Figure 10: Vacuum hold time chart relating geometry $\left(L, h_{c}, h_{s}\right)$ to material properties $(K)$ for the general case (top) and solved for Cycom 5320 plain weave (middle). The characteristic time (Eq. 26) relates panel geometry and material properties to vacuum hold time (bottom). 


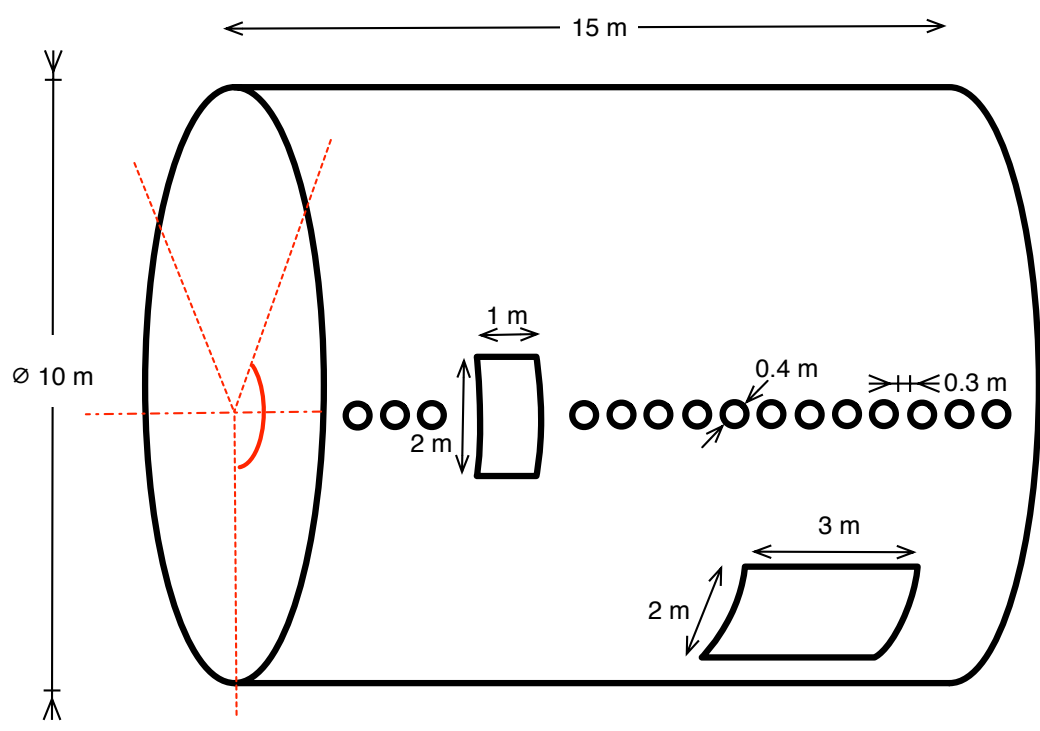

Figure 11: Geometry of a large fuselage structure case study. A full-barrel and third-barrel strategies are considered for air evacuation time. 


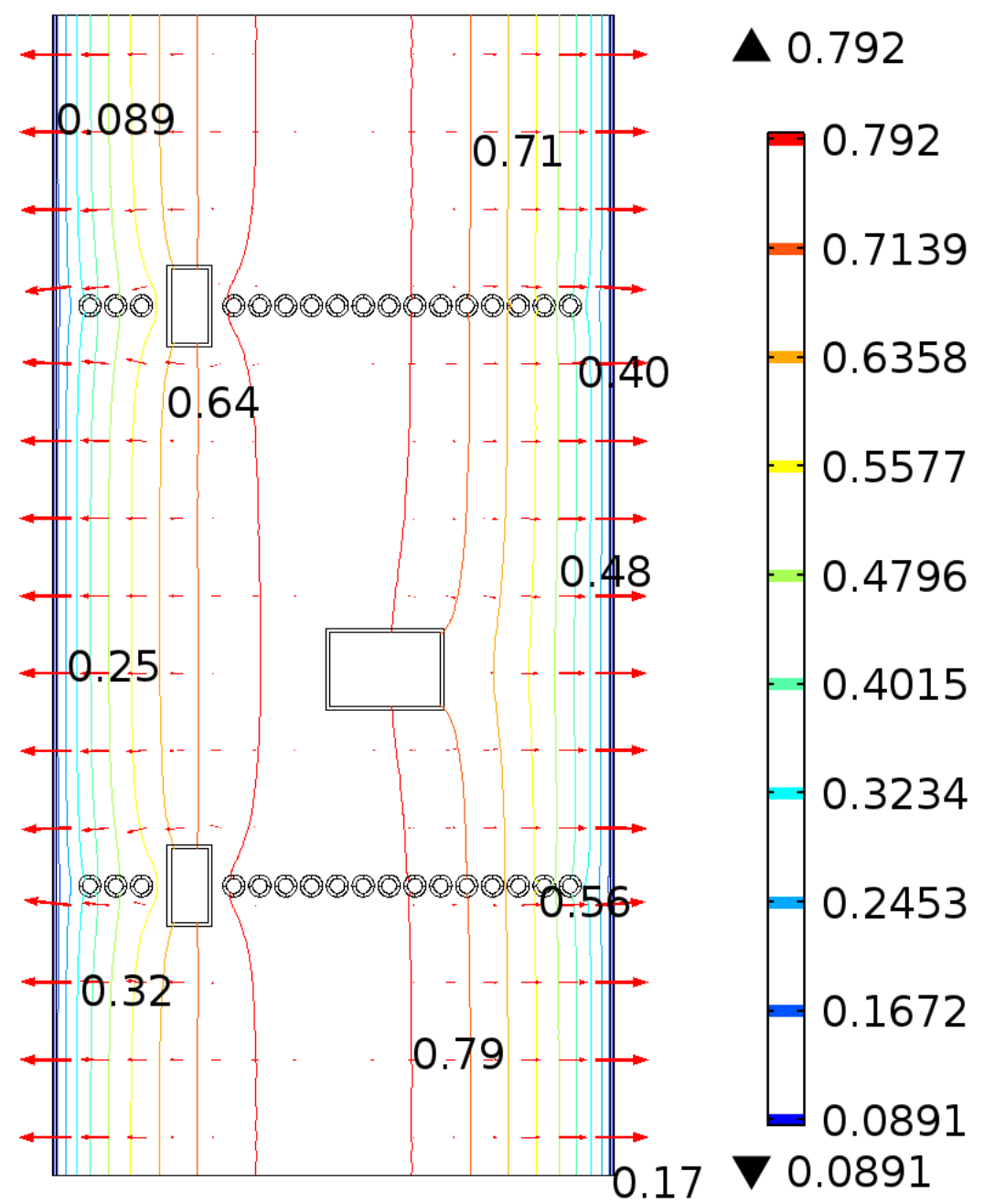

Figure 12: Predicted pressure $\left(10^{5} \mathrm{~Pa}\right)$ and velocity fields for the full-barrel configuration after $60 \mathrm{~h}$ into a vacuum hold. 


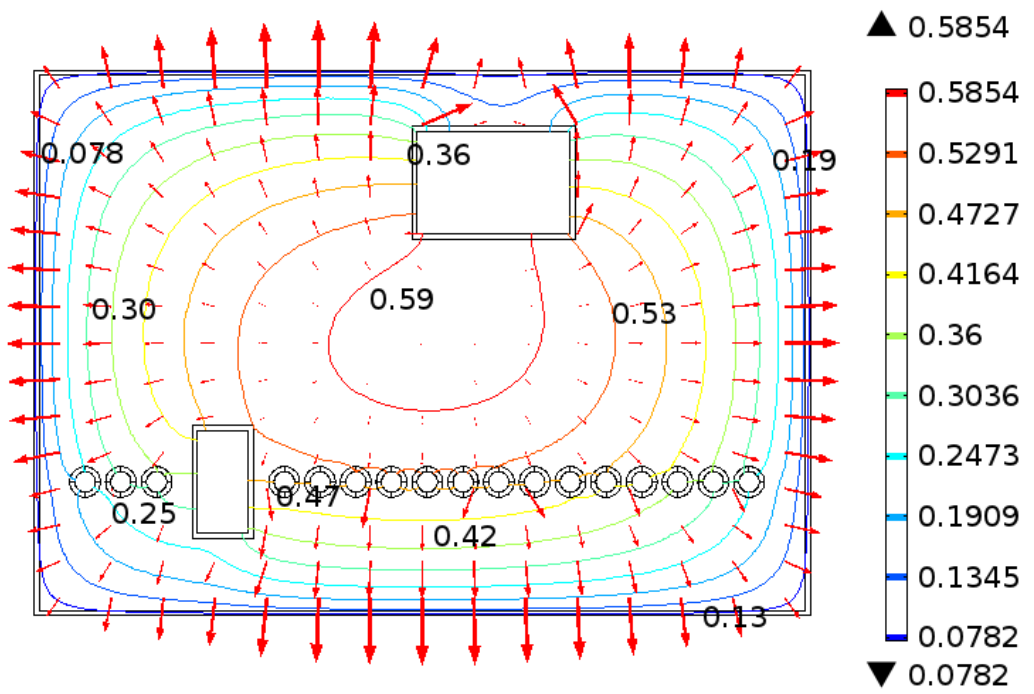

Figure 13: Predicted pressure $\left(10^{5} \mathrm{~Pa}\right)$ and velocity fields for the third-barrel configuration after $60 \mathrm{~h}$ into a vacuum hold.

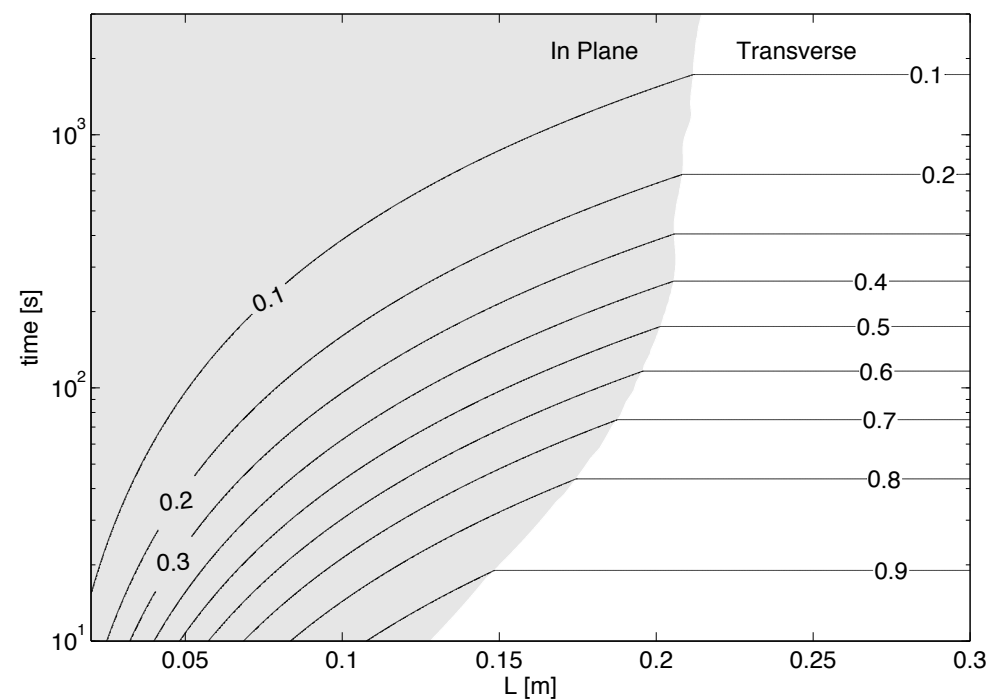

Figure 14: Iso-pressure in the part versus time and distance from edge breathing, for the non debulked Cycom 5320 case. The iso-values show that the pressure reduction is faster using a transverse breathing strategy for parts larger than $0.4 \mathrm{~m}(0.2 \mathrm{~m}$ to the centre of the panel). 


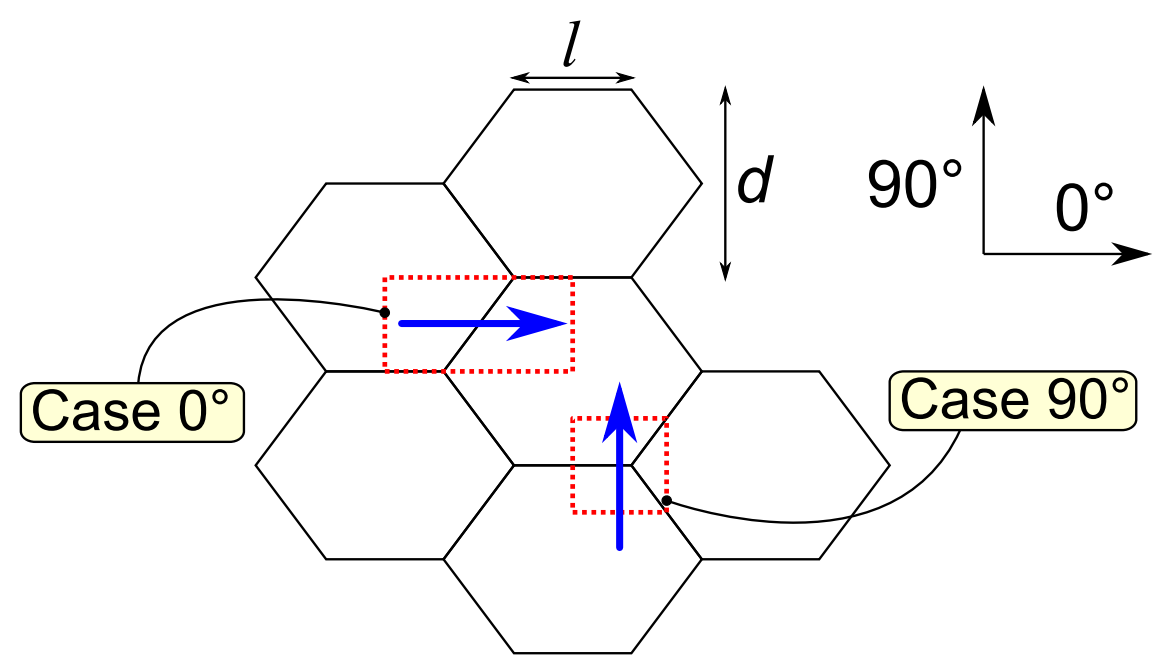

Figure 15: The two representative volume element considered to obtain the two principal direction equivalent permeabilities. 

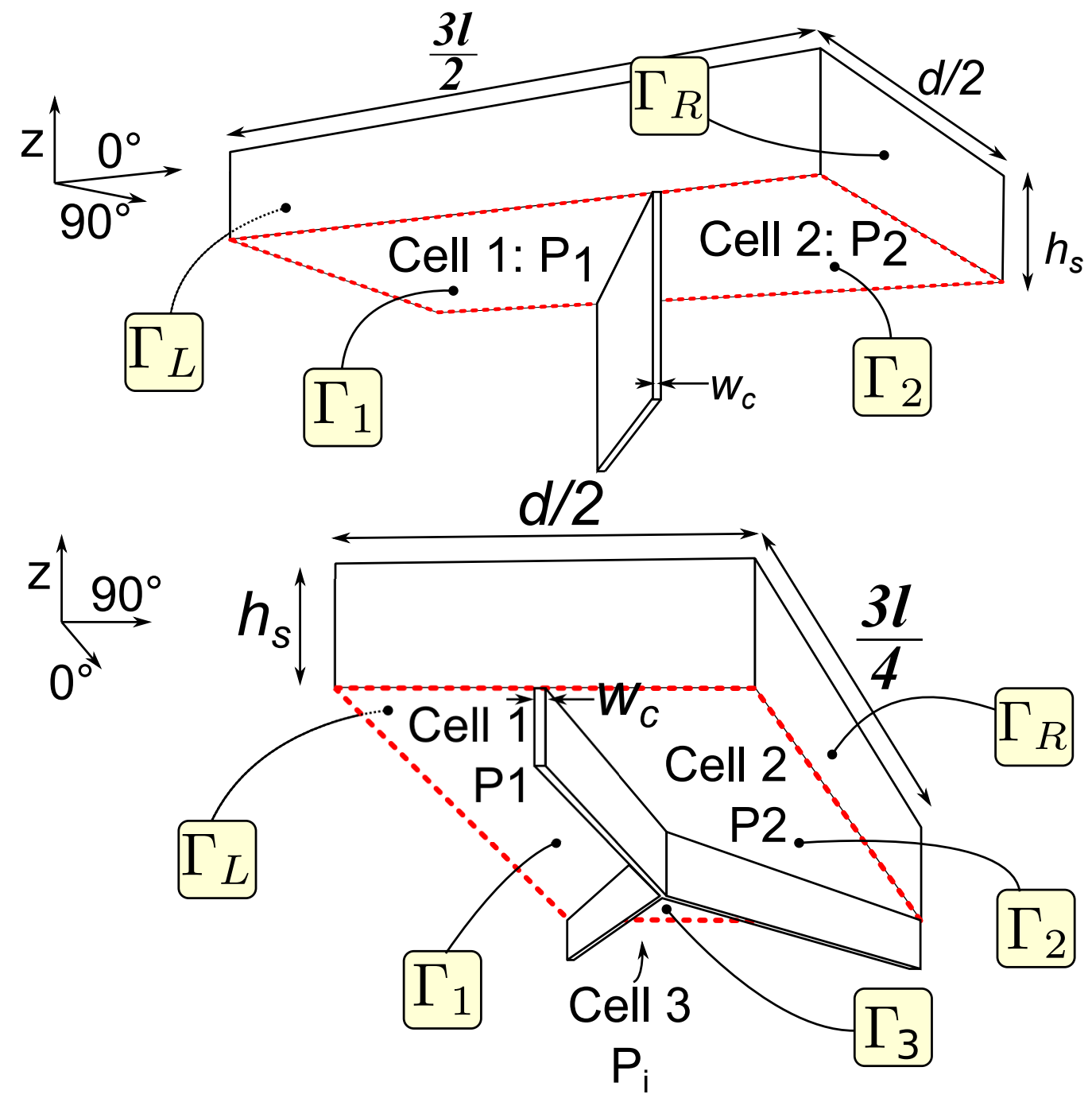

Figure 16: Representative volume element and boundary conditions. case $0^{\circ}$ (top) and case $90^{\circ}$ (bottom). 


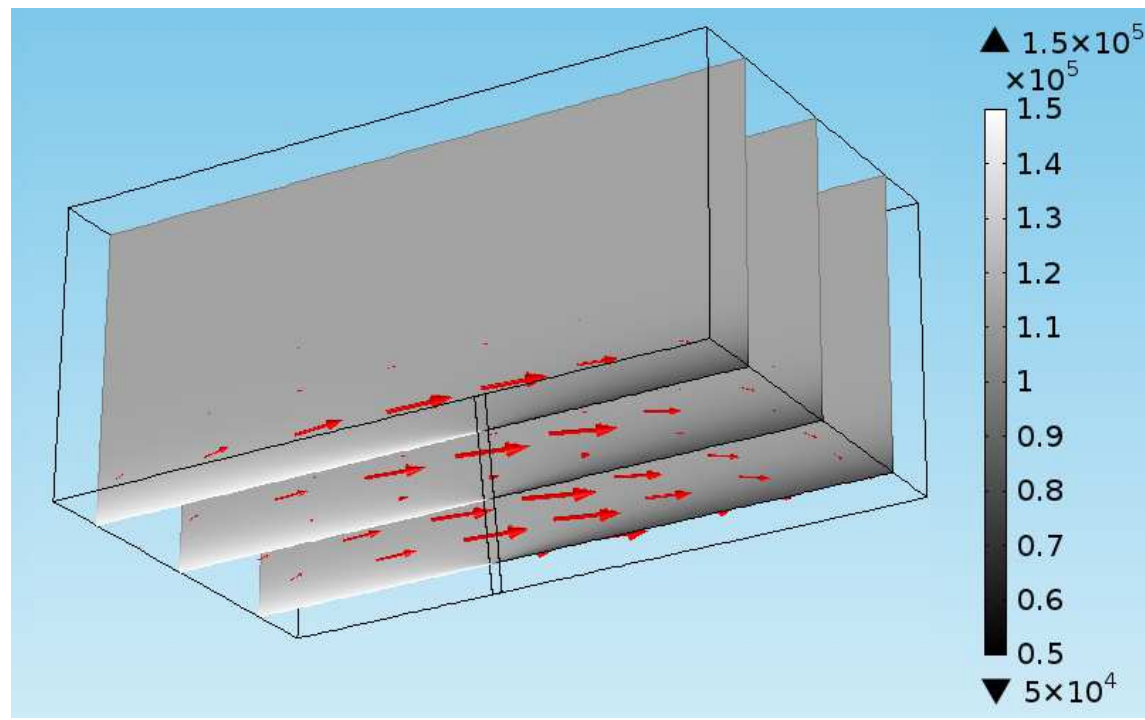

Figure 17: Numerical prediction of the mesoscopic pressure field for case $0^{\circ}(\mathrm{Pa})$

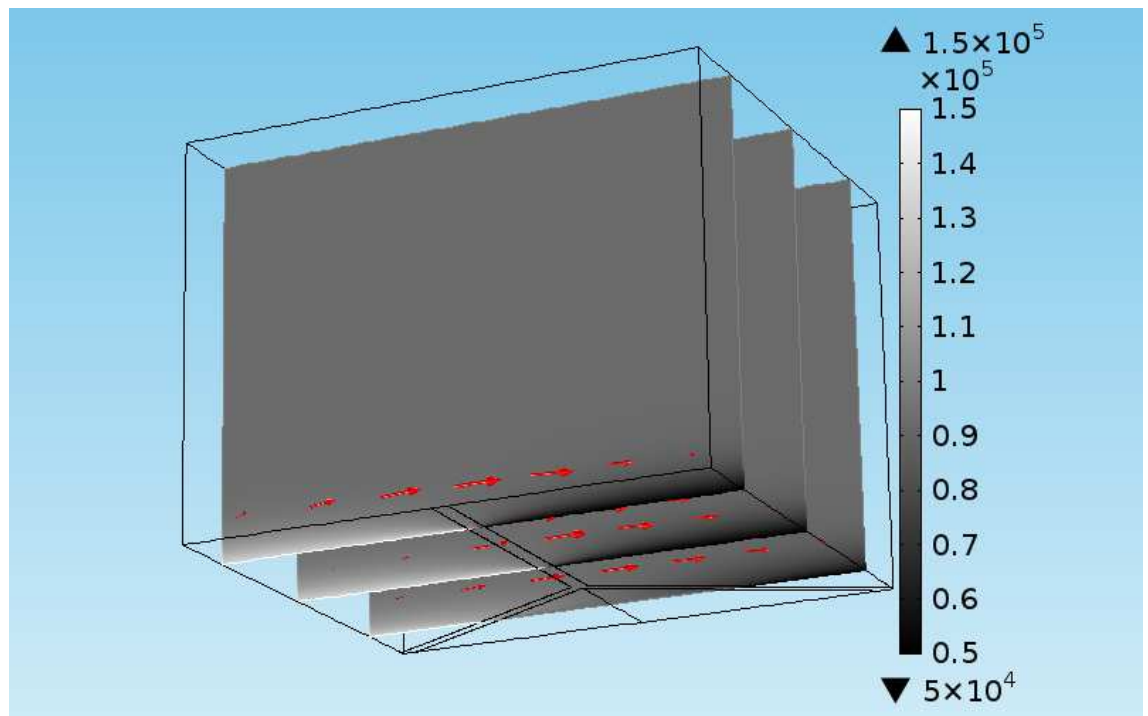

Figure 18: Numerical prediction of the mesoscopic pressure field for case $90^{\circ}(\mathrm{Pa})$. 
Table 1: Parameters used in the lab scale simulation.

\begin{tabular}{|c|c|c|}
\hline & & Non debulked debulked \\
\hline In-plane skin permeability $[6,28]$ & $K_{x}, K_{y}$ & $1.2 \times 10^{-13} \mathrm{~m}^{2} \quad 6 \times 10^{-14} \mathrm{~m}^{2}$ \\
\hline Prepreg porosity $[9]$ & $\phi$ & 0.244 \\
\hline Correction Factor & $\alpha$ & 0.32 \\
\hline Air viscosity & $\mu$ & $1.85 \times 10^{-5}$ Pa.s \\
\hline Core thickness & $h_{c}$ & $20 \mathrm{~mm}$ \\
\hline Chamfer angle & & $45^{\circ}$ \\
\hline Skin thickness & $h_{s}$ & $1.2 \mathrm{~mm}$ \\
\hline Edge band width & $e_{b}$ & $6.7 \mathrm{~cm}$ \\
\hline Sandwich length & $L$ & $0.9 \mathrm{~m}$ \\
\hline Sandwich width & $w$ & $0.3 \mathrm{~m}$ \\
\hline Atmospheric pressure & $\overline{P_{a t m}}$ & $1.013 \times 10^{5} \mathrm{~Pa}$ \\
\hline Vacuum pressure & $P_{v}$ & $5 \times 10^{3} \mathrm{~Pa}$ \\
\hline Time to pull vacuum & $t_{r a m p}$ & $10 \mathrm{~s}$ \\
\hline
\end{tabular}

Table 2: Parameters used for the large scale case study

\begin{tabular}{lcc} 
Core thickness & $h_{c}$ & $25 \mathrm{~mm}$ \\
Chamfer angle & & $45^{\circ}$ \\
Skin thickness & $h_{s}$ & $2.5 \mathrm{~mm}$ \\
Edge band width & $e_{b}$ & $10 \mathrm{~cm}$ \\
\hline
\end{tabular}

Table 3: Parameters used in the mesoscopic simulation.

Cell flat side $\quad d \quad 1.85 \mathrm{~mm}$

$\begin{array}{lll}\text { Cell diameter } & l & 3.2 \mathrm{~mm}\end{array}$

Skin thickness $\quad h_{s} \quad 1.2 \mathrm{~mm}$

Honeycomb wall thickness $\quad w_{c} \quad 50 \mu \mathrm{m}$

Pressure cell 1

$P_{1} \quad 1.5 \times 10^{5} \mathrm{~Pa}$

Pressure cell 2

\begin{tabular}{ll}
$P_{2}$ & $0.5 \times 10^{5} \mathrm{~Pa}$ \\
\hline
\end{tabular}

In plane permeability $\quad K_{x} \quad 6 \times 10^{-14} \mathrm{~m}^{2}$

Transverse permeability $[6,9,28] \quad K_{z} \quad 5 \times 10^{-17} \mathrm{~m}^{2}$ 